\title{
Fraud Deterrence in Dynamic Mirrleesian Economies*
}

\author{
Roc Armenter \\ F.R.B. Philadelphia
}

\author{
Thomas M. Mertens \\ NYU Stern
}

February 11, 2010

\section{PRELIMINARY DRAFT}

\begin{abstract}
Social and private insurance schemes rely on legal action to deter fraud and tax evasion. This observation guides us to introduce a random state-verification technology in a dynamic economy with private information. With some probability, an agent's skill level becomes known to the planner who prescribes a punishment if the agent is caught misreporting. We show how deferring consumption can ease the provision of incentives. As a result, the marginal benefit may be below the marginal cost of investment in the constrained efficient allocation suggesting a subsidy on capital. We characterize conditions such that the intertemporal wedge is negative in finite horizon economies. In an infinite-horizon economy, we find that the constrained efficient allocation converges to a high level of consumption, full insurance, and no labor distortions for any probablity of state-verification.
\end{abstract}

*We thank Cyril Monnet, Randy Wright, and the audiences in University of Maryland and Philadelphia Fed for comments and suggestions. The views expressed here do not necessarily reflect the views of the Federal Reserve Bank of Philadelphia or the Federal Reserve System. 


\section{Introduction}

Insurance is intended to provide support to people in adverse situations. An individual's condition determines the payment but is typically hard to monitor. Full insurance might not be optimal as agents struggle to resist the temptation of pocketing more support than they are entitled to. In a seminal contribution, Mirrlees (1971) formalized the trade-off between insurance and incentives by treating the agent's skills as private information. Subsequent work by Golosov, Kocherlakota and Tsyvinski (2003) extended the analysis of private information to dynamic economies. The optimal scheme in these economies is always designed such that agents pick the payments intended for them even if misreporting is undetectable.

Yet most of the insurance schemes we observe rely on the threat of legal action to induce truthful reporting. Laws against insurance and disability fraud are as commonplace as ancient. Of course, the rule of law requires to possess means of verifying the validity of claims. For example, insurance companies routinely inspect a subset of claimants after they filed for disability insurance. Similarly, elderly need to provide proof of eligibility for collecting on nursing care insurance, and the government routinely selects households for tax audits.

In this paper, we take a first step towards incorporating the threat of punishment as means of incentive provision in an optimal scheme. We do so by modifying an otherwise standard Mirrleesian economy. Idiosyncratic skill levels evolve over time following a stochastic process; output is given by a production function with constant returns to both labor and capital. The planner observes the amount each agent produces and consumes but, as in Mirrlees (1971), the effort exerted by the household is not observable. We study the constrained efficient allocations both in finite and infinite horizon economies.

In contrast to the standard Mirrleesian setup, the planner gets to verify the present skill level of a subset of agents selected each period at random. For the remaining households, the present skill level remains private information: the planner has no means to check past skill levels. Households must put forth their claims before it is known whether their skill level will be observable. This "random state verification" technology effectively assumes that governments and private companies can check the validity of some but not all claims; and whether a particular claim is observable or not is out of the control of both claimant and provider.

We find that the possibility of state verification reshapes the decisions and allocations on the equilibrium path. Agents' incentives to save are sensitive to state-verification: we find that the marginal benefit may be below the marginal cost of investment; and in infinite horizon economies

the constrained efficient allocation converges to full insurance, high consumption, and no labor distortions.

In equilibrium, no agent ever misreports the type. Not surprisingly then, the planner always 
prescribes the worst continuation welfare as a punishment to an agent found to be misreporting her type. Incentive provision improves with higher probabilities of state-verification and with sterner punishments. To focus on the equilibrium path, the worst continuation welfare, and thus punishment, is given by an exogenous lower bound on payoffs. Our setup encompasses the dynamic Mirrlees economy described in Golosov, Kocherlakota and Tsyvinski (2003) and the full information economy as special cases by setting the probability of state-verification to zero and high enough, respectively. Our interest lies in the intermediate values.

The key insight is that, with random state-verification, the provision of incentives can be made easier by increasing savings and deferring consumption to later periods. An agent caught misreporting forgoes the continuation welfare prescribed under truthful revelation: the effective punishment is given by the difference between the continuation welfare along the equilibrium path and the worst continuation welfare. The planner can make the effective punishment stronger, and thus ease the provision of incentives, by deferring consumption and welfare, which in turn demands increased savings.

Deferring consumption remains costly for the same reasons as in the Mirrleesian setting detailed in Golosov, Kocherlakota and Tsyvinski (2003). There are thus two counteracting forces regarding the intertemporal allocation of resources in the optimal contract. Which force dominates dictates whether the marginal benefit of investment is above or below the marginal cost, that is, whether the intertemporal wedge is positive or negative respectively and a capital tax or a subsidy would be needed to implemented the constrained efficient allocations. We illustrate both effects in a two-period economy.

An implied subsidy on capital is not just a mere possibility. Typically the sign of the intertemporal wedge will change across periods and across agents. We first discuss conditions for a negative intertemporal wedge in finite horizon economies. We show that the the marginal benefit of investment will be strictly above the marginal cost at some node provided the probability of state-verification is in a certain range. We also show that, for any positive probability of stateverification, there exists a long enough (but finite) time horizon such that the intertemporal wedge is non-positive.

The results are even starker for infinite horizon economies. We find that in the long-run almost all agents receive full insurance, a high level of consumption, and there are no distortions on the labor decision. In other words, all distortions vanish in the long run. Surprisingly, the result holds for any arbitrarily small probability of state-verification. We show how the planner can promise a high level of future consumption; high enough to make the effective punishment deter any misreporting even if full insurance is provided. ${ }^{1}$ Loosely speaking, the economy can "outgrow" the constraints imposed by private information. However, doing so is quite costly

\footnotetext{
${ }^{1}$ The assumption of constant returns to capital plays an important role here; ensuring that high consumption is indeed feasible.
} 
as the economy needs to accumulate aggregate capital and incur in low consumption along the transition path. Nevertheless convergence to full insurance occurs with probability one. The rationale for deferring consumption and welfare eventually dominates in an infinite-horizon economy as all agents will experience a (long enough) streak of high skill level realizations. Along with these positive shocks, it becomes easier and easier to provide incentives with future consumption: eventually the effective punishment is strong enough to support full insurance, which becomes an absorbing state. Our result here makes contact with those in Albanesi and Armenter (2008): as long as it is possible to front-load all the distortions present in the economy, the constrained efficient allocation rules out permanent intertemporal distortions.

We also find that agents whose skill level is observable are rewarded with higher consumption and leisure as long as incentive-compatibility constraints are binding. Thus it is optimal to introduce some consumption uncertainty linked to the possibility of state-verification. In infinite horizon economies, though, the rewards are eventually phased out.

In contrast with the savings decisions, the prescriptions regarding the labor supply are robust to the introduction of the random state verification. As in the standard Mirrleesian framework, the labor decision of the agent with the top skill level is left undistorted, while the output of other agents is taxed. The exception is that the labor supply decision of agents whose skill level has been verified always remains undistorted.

Our work relates to a literature on the optimal taxation of savings. Early work on economies with linear factor taxation finds a zero tax rate on capital income, see Judd (1985) and Chamley (1986). Subsequent research has further explored the Mirrlees approach to dynamic optimal taxation and disability insurance. ${ }^{2}$

There is a limited literature on the design of audit mechanisms in private information economies. Border and Sobel (1987) characterize an optimal audit mechanism design for an economy in which agents have private information on their endowment. Cremer and Gahvari (1995) analyze the implications of an audit scheme for static private information economies.

The paper is structured as follows. Section 2 presents a simple economy with just two periods that illustrates our results concerning the incentives to save and the allocation of consumption and output across agents. Section 3 lays out the set-up for the general economy. We define and characterize the constrained efficient allocation in Section 4. We start with our formal results on the allocation of consumption and output across agents in Section 5, and follow with our results for the incentives-to-save in Section 6. Section 7 documents our findings on the long-run properties of the constrained efficient allocation. Section 8 concludes.

\footnotetext{
${ }^{2}$ A necessarily incomplete list in a rapidly increasing literature is Albanesi and Sleet (2006), Kocherlakota (2005), Golosov and Tsyvinski (2006), Werning (2007), and Farhi and Werning (2007).
} 


\section{Setup and Constrained-Efficient Allocations}

\subsection{Setup}

Time is discrete $t=0,1, \ldots, T$ with $T$ possibly being infinite. The economy is populated by a continuum of infinitely lived, ex-ante identical agents. Agents are subject to idiosyncratic shocks to their skill levels. At each date $t$, the agent's skill $\theta_{t}>0$ takes on a value from a finite support $\Theta$ according to the probability distribution $\{\pi(\theta): \theta \in \Theta\}$. Let $\theta^{t}=\left\{\theta_{0}, \theta_{1}, \ldots, \theta_{t}\right\}$ be the history of skill realizations and $\Theta^{t}$ the corresponding support for histories up to date $t$. The skill level of an agent determines the labor effort $l_{t}$ needed to produce output $y_{t}=\theta_{t} l_{t}$. We assume that skill levels are independent across agents and the law of large numbers applies. We denote with $\pi\left(\theta^{t}\right)$ both the fraction of agents with history $\theta^{t}$ and the probability for of such event.

All agents derive utility from consumption $c_{t} \in \mathbb{R}_{+}$according to the utility function $u$ : $\mathbb{R}_{+} \rightarrow \mathbb{R}$ and disutility from labor $l_{t} \in \mathbb{R}_{+}$according to $v: \mathbb{R}_{+} \rightarrow \mathbb{R}$. We find it useful to express the per-period payoff in terms of output rather than labor. Let the consumption-output bundle for an agent be $x_{t}=\left\{c_{t}, y_{t}\right\} \in \mathbb{R}_{+}^{2}$, then her utility is given by

$$
h\left(x_{t} ; \theta_{t}\right)=u\left(c_{t}\right)-v\left(\frac{y_{t}}{\theta_{t}}\right)
$$

where $h: \mathbb{R}_{+}^{2} \times \Theta \rightarrow \mathbb{R}$.

Relative to the literature on Mirrleesian economies, the key innovation of this paper is the possibility that the skill level becomes observable to a planner. An exogenous process determines whether the skill level of an agent is only privately known or observable. Let $a_{t}$ be an i.i.d. random variable which takes the value $a_{t}=1$ with probability $p$ and $a_{t}=0$ otherwise. At every date and for every agent, variable $\alpha_{t}=a_{t} \theta_{t}$ is observable: however, if $\alpha_{t}=0$ then the variable is uninformative about the skill level. ${ }^{3}$ Let $a^{t}$ be the history of realizations and $A^{t}=\{0,1\}^{t}$ the corresponding support. Note that only the skill level at the current date is revealed in a given period. In terms of agents' decisions, we assume that consumption and output are observable, but the exerted effort is not.

Each agent's type consists of the history of skills and state verifications $s^{T}=\left\{\theta^{T}, a^{T}\right\} \in$ $\Theta^{T} \times A^{T} \equiv S^{T}$. From the probability process for skills and the probability of state verification, we can define a probability over the set of types $S^{t}$ for $t=0,1, \ldots, T$ which we denote with $\pi\left(s^{t}\right)$ in a slight abuse of notation.

\footnotetext{
${ }^{3}$ This specification is a convenient shortcut for notation but requires that the uninteresting case of $\theta \neq 0$ is ruled out. Equivalently, we can let $\alpha$ be a random variable with values in $\Theta \cup\{\varnothing\}$. For each agent, $\alpha_{t}$ equals her skill level $\theta_{t}$ with probability $p$ and it is uninformative, $\alpha_{t}=\{\varnothing\}$, with probability $1-p$.
} 
Let $x$ denote a mapping that assigns each type in $S^{T} \rightarrow \mathbb{R}_{+}^{2 T}$ consumption and labor allocations at every date. Agents have von Neumann-Morgenstern preferences and value a plan $x$ according to

$$
W_{0}(x)=\sum_{t=0}^{T} \sum_{s^{t} \in S^{t}} \beta^{t} \pi\left(s^{t}\right) h\left(x\left(s^{t}\right) ; \theta_{t}\right)
$$

where $\beta \in(0,1)$ is the time discount factor. As part of the setup, we assume that period utility cannot drop below a lower bound $v$, i.e. $h\left(x\left(s^{j}\right) ; \theta_{j}\right) \geq v$. It turns out to be more convenient to translate this lower bound on utility into a the lower bound on continuation welfare. For all dates and all type realizations $s^{t} \in S^{t}$,

$$
\sum_{j=t}^{T} \sum_{s^{j} \in S^{j}} \beta^{j-t} \pi\left(s^{j} \mid s^{t}\right) h\left(x\left(s^{j}\right) ; \theta_{j}\right) \geq\left(1-\beta^{T+1-t}\right) \Upsilon
$$

where $\Upsilon=v(1-\beta)^{-1}$ and $\pi\left(s^{j} \mid s^{t}\right)$ is the conditional probability of history $s^{j}$ given $s^{t}$. The lower bound on welfare plays an important role in our analysis as it determines the maximum punishment a planner can prescribe for a deviant agent.

We list below all the assumptions on preferences. In addition to the standard convexity and differentiability properties, we assume a particular set of boundary conditions that allow us to focus on interior allocations. The boundary conditions also ensure that the lower bound on welfare (1) can be attained with an allocation in the positive quadrant.

\section{Assumption 1 (Preferences)}

Preferences are such that

1. (Monotonicity) Both $u(c)$ and $v(l)$ are strictly increasing,

2. (Differentiability) Both $u(c)$ and $v(l)$ are twice differentiable,

3. (Convexity) Utility function $u(c)$ is strictly concave and $v(l)$ is strictly convex,

4. (Boundary conditions) Utility function $u(c)$ is unbounded above and below; and $v(l)$ is bounded below and satisfies $v^{\prime}(0)=0$.

Assumption 1.4 ensures that equation (1) specifies the effective lower bound. The disutility of labor is bounded from below and hence we can normalize it to $v(0)=0$ without loss of generality.

An allocation is a pair $\{x, K\}$ where $K=\left\{K_{t+1}\right\}_{t=0}^{T}$ is a non-negative sequence specifying aggregate capital at all dates $t=1,2, \ldots, T$. The initial level of capital $K_{0}>0$ is taken as given. A feasible allocation satisfies the lower bound on welfare (1) for all $s^{t} \in S^{t}$ and the resource constraint at all dates $t$,

$$
C_{t}+K_{t+1} \leq R K_{t}+Y_{t}
$$


where $R=\beta^{-1}$ denotes the gross interest rate, and $C_{t}$ and $Y_{t}$ stand for aggregate consumption and output respectively. ${ }^{4}$ The production function displays constant marginal returns to capital. We will return to this assumption later in the paper as it plays an important role for some of our long-run results.

\subsection{Efficient and constrained-efficient allocations}

Before discussing the implications of information frictions, we define the best allocation only subject to feasibility. We denote such an allocation as "efficient" or "first best" to distinguish it from "constrained efficient" or "second best" allocations to be defined later.

\section{Definition 1 (Efficient allocation)}

A feasible allocation $\{x, K\}$ is efficient if

$$
W_{0}(x) \geq W_{0}\left(x^{\prime}\right)
$$

for all feasible allocations $\left\{x^{\prime}, K^{\prime}\right\}$.

Condition (1) implies a lower bound on $W_{0}$. To guarantee that the objective function $W_{0}$ is also bounded from above, we need some additional conditions on preferences. We therefore adopt a standard assumption used for economies with constant marginal returns to capital. The assumption encompasses several utility function specifications, including logarithmic and constant elasticity of substitution (CES) utility functions.

\section{Assumption 2}

There exists scalars $a, b$ and $\gamma<1$ such that $u(c) \leq a+b c^{\gamma}$ for all $c \geq 0$.

This assumption ensures that the objective function is finite for the set of feasible allocations. Consequently, we can characterize the efficient and constrained efficient allocations as optimal control problems.

Let us now turn to analyzing the set of feasible allocations that can be implemented given the presence of private information. We start by being precise about how information is revealed and communicated to agents and planner alike. At the beginning of each period $t$, the skill level $\theta_{t}$ becomes known only to the agent. The agent then submits a report to the planner,

\footnotetext{
${ }^{4}$ Aggregate consumption and output are the sum over individual consumption and output weighted by the mass of people:

$$
\begin{aligned}
C_{t} & =\sum_{s^{t}} \pi\left(s^{t}\right) c\left(s^{t}\right) \\
Y_{t} & =\sum_{s^{t}} \pi\left(s^{t}\right) y\left(s^{t}\right) .
\end{aligned}
$$
}


$\sigma_{t}\left(s^{t-1}, \theta_{t}\right) \in \Theta$. The state-verification variable $a_{t}$ is realized only after the report has been filed. The planner then dictates a consumption and output allocation given both the report, the realization of state verification $a_{t}$, and, if the skill level is revealed, the true type of the agent. We allow the planner to specify different allocations for an agent depending on whether the skill level is public information even if the agent is reporting truthfully.

Formally, we define a reporting strategy as a mapping $\sigma_{t}: S^{T} \rightarrow \Theta^{T}$ such that for all dates $t \geq 0$, the report $\sigma_{t}$ is measurable with respect to $\left\{s^{t-1}, \theta_{t}\right\}$. The truth-telling reporting strategy $\sigma^{*}$ is given by $\sigma_{t}^{*}\left(s^{t-1}, \theta_{t}\right)=\theta_{t}$ for all realizations in $S^{T}$. Let $\Sigma$ be the set of reporting strategies. A contract $\xi$ specifies consumption and output at all dates given history $s^{t-1}$, report $\hat{\theta}_{t}$, and observable variable $\alpha_{t}=a_{t} \theta_{t} .{ }^{5}$ Let $\xi(\sigma)$ be the allocation plan spanned by the reporting strategy $\sigma$.

A feasible allocation $\{x, K\}$ is admissible if $\xi\left(\sigma^{*}\right)=x$ for some contract $\xi$ such that

$$
W_{0}\left(\xi\left(\sigma^{*}\right)\right) \geq W_{0}(\xi(\sigma))
$$

for all $\sigma \in \Sigma$. That is, the truth-telling strategy is optimal for all agents. We now use this language to define a constrained efficient allocation.

\section{Definition 2 (Constrained-efficient allocation)}

An admissible allocation $\{x, K\}$ is constrained efficient if

$$
W_{0}(x) \geq W_{0}\left(x^{\prime}\right)
$$

for all admissible allocations $\left\{x^{\prime}, K^{\prime}\right\}$.

\subsection{Characterization of constrained-efficient allocations}

We seek to characterize constrained-efficient allocations as a solution to an optimal control problem. The first step is to write the incentive-compatibility constraints in terms of the allocation plan. This task is simplified by two well-known results. First, we only need to consider one-step deviations from the truth-telling strategy. Second, we can write the incentive-compatibility constraint knowing that the planner prescribes the lowest continuation welfare possible whenever an agent is caught misreporting her skill level. The reason is that no agent will actually misreport on the equilibrium path: to prescribe the maximum punishment available in such events comes at no cost and improves incentives on the equilibrium path.

We simplify further the formulation of the incentive-compatibility constraints by keeping

\footnotetext{
${ }^{5}$ Note that a contract and allocation are different mappings, as a contract must be measurable over the support for the report and observable variable $\alpha_{t}$ in addition to the actual skill level.
} 
track of promised utility. Let $z\left(s^{t}\right)$ be the expected continuation utility for date $t+1$ after history $s^{t}$. We state our promise-keeping constraint to make the definition of $z\left(s^{t}\right)$ explicit,

$$
z\left(s^{t}\right)=\sum_{s^{t+1} \in S^{t+1}} \pi\left(s^{t+1} \mid s^{t}\right)\left\{h\left(x\left(s^{t+1}\right) ; \theta_{t+1}\right)+\beta z\left(s^{t+1}\right)\right\}
$$

for all histories $s^{t} \in S^{t}$ for dates $t \geq 0$. If $T$ is finite, the terminal condition, $z\left(s^{T}\right)=0$, applies to all histories $s^{T} \in S^{T}$, as part of the set of promise-keeping constraints. These constraints allow us to rewrite the set of admissible allocations in a different form. Using the promised utility, we can express truthful reporting in a single constraint for each type and period. A feasible allocation $\{x, K\}$ is admissible if and only if the following incentive compatibility constraint is satisfied

$$
\sum_{a_{t} \in\{0,1\}} \pi\left(a_{t}\right)\left\{h\left(x\left(s^{t}\right) ; \theta_{t}\right)+\beta z\left(s^{t}\right)\right\} \geq p\left(1-\beta^{T+1-t}\right) \Upsilon+(1-p)\left\{h\left(x\left(\hat{s}^{t}\right) ; \theta_{t}\right)+\beta z\left(\hat{s}^{t}\right)\right\}
$$

at all dates $t \geq 0$, for all $\left\{s^{t-1}, \theta_{t}\right\} \in S^{t-1} \times \Theta$ and all skill levels $\theta^{\prime} \in \Theta$, where $\hat{s}^{t}=\left\{s^{t-1}, \theta^{\prime}, 0\right\}$. Note that there is no separate incentive-compatibility constraints for $a_{t}=0$ and $a_{t}=1$ : the agent must submit the report before knowing the realization of $a_{t}$. Thus the value of truthful reporting and misreporting is given by a lottery spanned by the realization of the state-verification variable $a_{t}$.

We characterize constrained efficient allocations as solutions to the following optimal control problem

$$
\sup _{\{x, K, z\}} W_{0}(x)
$$

subject to the resource constraint (2) at all dates $t=0,1, \ldots, T$, and to the promise-keeping and incentive-compatibility constraints (3) and (4) at all nodes $s^{t} \in S^{t}$, as well as non-negativity constraints and the lower bound constraint on continuation welfare (1).

The state-verification technology relaxes the incentive-compatibility constraints, as can be seen from equation (4) and thus enlarges the set of admissible allocations. Before we proceed further, we ask under which conditions the efficient allocation is admissible, rendering trivial the problem of constrained efficiency. From the incentive-compatibility constraint (4) the role of the lower bound on welfare, $v$, becomes clear. If welfare were unbounded below, the planner could prescribe an infinite punishment in the event of misreporting: the efficient allocation would then trivially satisfy the incentive-compatibility constraints. We shall treat the lower bound $v$ as a fixed parameter for the remainder of the paper.

The next assumption ensures that the lower bound is low enough such that the set of admissible allocations exists and is not a singleton. To ensure that the lower bound can be achieved 
with a feasible allocation, we also assume $\Upsilon$ is finite.

\section{Assumption 3}

The lower bound $v$ is set in a way that the set of admissible allocations has a non-empty interior and $v>-\infty$.

Taken the punishment level as given, we now ask about conditions on the state-verification probability such that the efficient allocation is admissible. Clearly, the efficient allocation is not implementable for a sufficiently low state-verification probability $p$. For the case where the probability of state verfication is zero, we retrieve the standard dynamic Mirrlees formulation. In that case the efficient allocation is never admissible. On the other end, if $p$ is large enough, the efficient allocation satisfies the incentive-compatibility constraints (4). Indeed, there exists a threshold $p^{*}$ such that the set of admissible allocations for $p<p^{*}$ does not contain the efficient allocation.

\section{Lemma 1}

There exists $p^{*} \in(0,1)$ such that the efficient allocation is not admissible if and only if $p<p^{*}$.

\section{A Simple Two-Period Economy}

We use a two-period economy to illustrate the mechanics of the setup. We are interested in how exogenous state-verification shapes the incentives to save and work under the constrained efficient allocation.

Therefore we use the setup of the previous section with only two periods as a special case. For illustrative purposes, we do not allow the planner to give out rewards for truth-telling. At time $t=1$, each agent chooses how much of her initial endowment to consume, $c_{1}$, leading to an amount of savings $s=y_{1}-c_{1}>=0$. All agents behave symmetrically in the first period since there is no source of heterogeneity.

In the second period agents learn their idiosyncratic skill level. Half of the agents are able to produce one unit of output with $1 / \theta$ units of effort where $\theta>1$. The other half produces only one unit of output for each unit of effort. The resource constraint at date $t=2$ is given by

$$
\frac{1}{2}\left(c_{2}(\theta)-y_{2}(\theta)\right)+\frac{1}{2}\left(c_{2}(1)-y_{2}(1)\right) \leq s .
$$

where $\left\{c_{2}(\theta), y_{2}(\theta)\right\}$ and $\left\{c_{2}(1), y_{2}(1)\right\}$ denote consumption and output produced at date $t=2$ for agents with skill level $\theta$ and 1 respectively. For simplicity, we assume that the return to savings is 1 . A feasible allocation $x$ is a vector specifying consumption at date $t=1$, savings, and consumption and output at both dates as a function of the skill-level such that the resource constraint is satisfied at both dates. 


\subsection{Constrained Efficient Allocation}

The incentive-compatibility constraints do not reflect any promised utility since we only set up a two period economy. Hence we can write them as

$$
u\left(c_{2}(\theta)\right)-v\left(y_{2}(\theta) / \theta\right) \geq p v+(1-p)\left(u\left(c_{2}(1)\right)-v\left(y_{2}(1) / \theta\right)\right)
$$

where $v$ stands for the amount of utility agents receive if caught misreporting. The incentive compatibility constraint ensures that the high-skill level reveals her type before knowing whether it will be observable. As in the standard Mirrlees problem, the corresponding incentivecompatibility constraint for the low skill agent is automatically satisfied.

The possibility of the skill level becoming public information relaxes the incentive compatibility constraint. The higher the probability of observing the true type and the more severe the punishment, the easier it is to provide incentives. Indeed, if $v$ is low enough or $p$ is sufficiently close to 1 , the first best allocation becomes incentive compatible. On the other end, if skills are never verified, we recover the standard formulation of the dynamic Mirrleesian setup.

To arrive at the constrained efficient allocations, we maximize social welfare

$$
u\left(c_{1}\right)-v\left(y_{1}\right)+\frac{1}{2}\left(u\left(c_{2}(\theta)-v\left(\frac{y_{2}(\theta)}{\theta}\right)\right)+\frac{1}{2}\left(u\left(c_{2}(1)-v\left(y_{2}(1)\right)\right)\right.\right.
$$

subject to the incentive compatibility constraint (5) and the resource constraint.

We are interested in the constrained efficient allocation, that is, the incentive-compatible allocation attaining the highest welfare. From the first-order conditions of the optimal control problem, we derive a relation between the consumption levels of the two different types in the second period:

$$
u_{2}^{c}(\theta)(1+\mu)=u_{2}^{c}(1)(1-(1-p) \mu)
$$

where $\mu$ is the Lagrangian multiplier associated with the incentive compatibility constraint. ${ }^{6}$ As long as the first best allocations are not incentive compatible, that is $\mu>0$, consumption is not equated across agents. More precisely, the agent of type $\theta$ has a higher level of consumption, as in the standard Mirrlees problem.

We start by exploring how private information and the state verification technology shape agents' incentives to work. We find that the prescriptions of the static Mirrlees problem are remarkably robust. For any state-verification probability, the marginal rate of substitution is

\footnotetext{
${ }^{6}$ We have normalized the Lagrangian multiplier $\mu$ by the measure of agents of type $\theta$.
} 


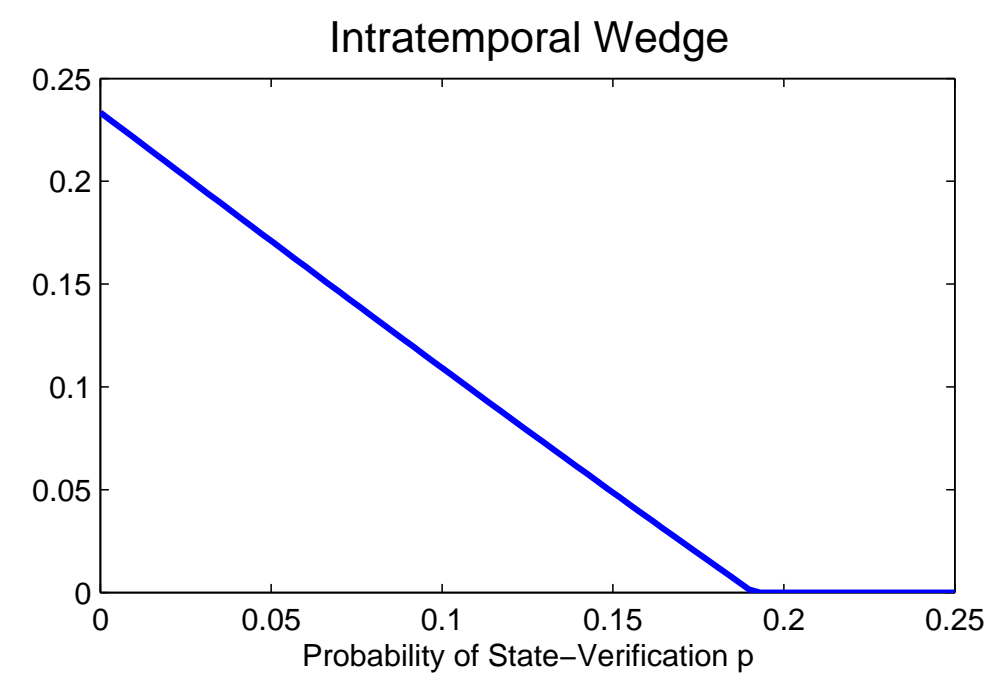

Figure 1: Implied labor tax rate for various probabilities of state verification.

equated to the marginal rate of transformation for the agent with the highest skill.

$$
u^{\prime}\left(c_{2}(\theta)\right)=\frac{1}{\theta} v^{\prime}\left(\frac{y_{2}(\theta)}{\theta}\right)
$$

Thus the implied marginal labor wedge is zero for the agent with high skills as in Mirrlees (1971). As long as the first-best allocation is not incentive compatible, the allocations suggest a positive labor tax for low skill types.

$$
v^{\prime}\left(y_{2}(1)\right)\left(1-(1-p) \mu \frac{v^{\prime}\left(y_{2}(1) / \theta\right.}{\theta v^{\prime}\left(y_{2}(1)\right)}\right)=(1-(1-p) \mu) u^{\prime}\left(c_{2}(1)\right)
$$

Both results follow immediately from the first-order conditions for consumption and output.

The probability of state-verification $p$ influences the magnitude of the labor wedge of the constrained efficient allocation. It is easier to provide incentives as $p$ increases, and the optimal labor wedge is decreasing. Figure 1 plots the optimal labor wedge for the low skill agent as a function of $p \cdot{ }^{7}$ Labor is undistorted only when the probability of state-verification is high enough that the incentive-compatibility constraint is no longer binding. In other words, labor distortions only disappear when the first best allocation is incentive-compatible. We show these results for the general case with multiple types and many periods in section 4 .

Let us now sign the intertemporal wedge for the optimal allocation. The question here is whether the intertemporal distortion suggests a tax or subsidy for capital. By manipulation

\footnotetext{
${ }^{7}$ Agents value allocations according to preferences that satisify the assumptions of the previous section. For our simple economy, we choose utility of consumption $u(c)=\log (c)$ and disutility of labor $v(l)=-l^{4} / 4$ with parameter choices $\theta=2$ and $v=-1 / 4$.
} 
of the first-order conditions derived from the maximization problem above, we arrive at an intertemporal optimality condition given by

$$
E\left\{\frac{1}{u_{2}^{c}}\right\}=\frac{1}{u_{1}^{c}}\left(1+\frac{p \mu}{2}\right)
$$

In the special case where types are never verified, equation (6) encompasses the well-known inverse Euler equation,

$$
u_{1}^{c}=\left[E\left\{\frac{1}{u_{2}^{c}}\right\}\right]^{-1} .
$$

In this case, there is always variation in consumption across types. Jensen's inequality then implies $u_{1}^{c}<E\left\{u_{2}^{c}\right\}$. The intertemporal wedge must be positive in order to implement the constrained efficient allocations. This result carries on for infinite horizon economies with arbitrary stochastic processes for the agents' type.

Now consider the possibility of exogenous state-verification, $p>0$. As long as $p \mu>0$ in equation (6), we cannot guarantee the above result of a positive implied savings tax.

Fortunately, it is still possible to sign the term $u_{1}^{c}-E\left\{u_{2}^{c}\right\}$. Using the first-order conditions for $c_{2}(\theta)$ and $c_{2}(1)$, we can solve for $E\left\{u_{2}^{c}\right\}$ in terms of $\mu$ and $p$ which in turn implies $u_{1}^{c} \geq E\left\{u_{2}^{c}\right\}$ whenever

$$
\mu \leq \frac{1}{2} \frac{p}{1-p}
$$

Moreover, $u_{1}^{c}=E\left\{u_{2}^{c}\right\}$ if and only if $\mu=0$ or (7) holds with strict equality. It is possible to express condition (7) in terms of a threshold $\hat{p}$ for the probability of state-verification at which the equality is satisfied. Heuristically, the Lagrangian multiplier $\mu$ is decreasing in $p$ as the incentive-compatibility constraints become relaxed; and the right hand side of (7) is increasing in $p$. We derive the general version of this inverse Euler equation for our setup in section 5 .

We thus find an implied subsidy if it is likely enough to observe the skill level, $p \geq \hat{p}$. Figure 2 displays the implied savings tax needed to implement the constrained efficient allocation for different state-verification probabilities. The comparative statics for the implied tax are not monotone and lead to a sign-switch: around $p=.05$ the optimal saving tax turns into a subsidy. The exact value of the threshold depends on the primitives of the economy - the curvature of the preferences, the dispersion in skills, as well as the lower bound on welfare. Importantly though, the first best allocations are not incentive compatible at $p=\hat{p}$.

Before interpreting our results, let us first revisit briefly why the dynamic Mirrlees problem calls for savings to be taxed. The incentive-compatibility constraints require some spread between the consumption of high and low skill level agents. The more resources are available in the economy, the larger the spread must be. In other words, savings make the provision 


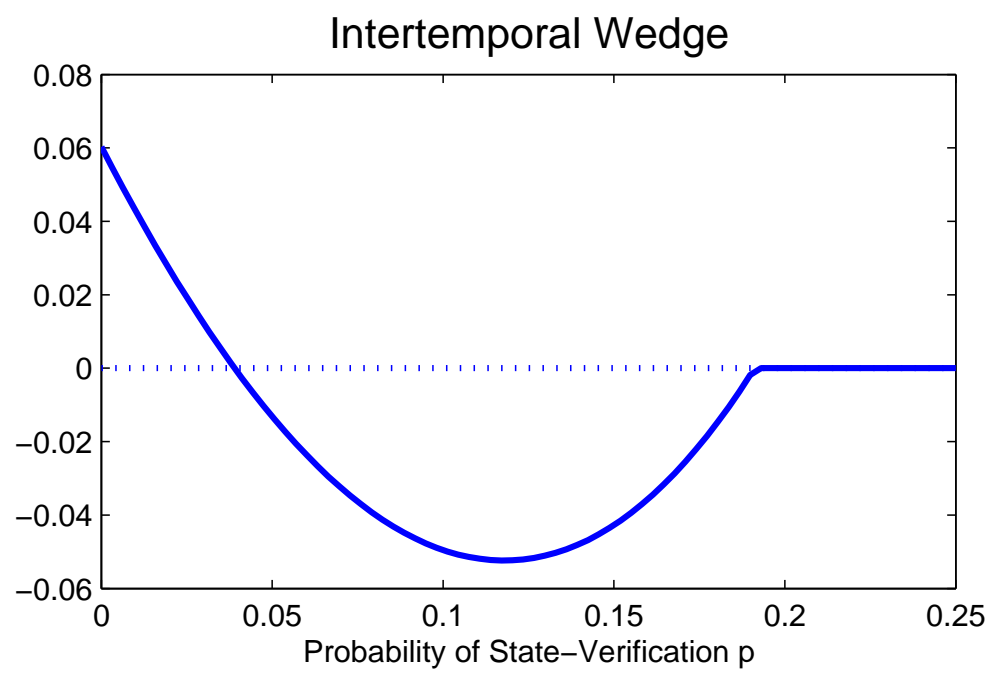

Figure 2: Implied savings tax rate for various probabilities of state verification.

of incentives more difficult. As a result, consumption and welfare are frontloaded under the constrained efficient allocation.

The possibility of observing the agent's type ex-post opens a new channel to improve incentives. State-verification allows to prescribe a punishment for any agent caught misreporting the skill level. Since this punishment is never administered on the equilibrium path, the planner equates the welfare of any misreporting agent to the lower bound on welfare. Everything else constant, the higher expected consumption for truthful reporting, the sterner the punishment for misreporting, and thus the more effective is state-verification in relaxing the incentive-compatibility constraints. Hence, additional resources, and thus savings, can improve the provision of incentives by raising overall welfare.

In short, savings can help the provision of incentives in the presence of state verification. Whether savings are taxed or subsidized depends, ultimately, on which effect dominates. Indeed, there is a probability of state verification such that the implied tax on savings is negative. We discuss the incentives to save in more detail in section 5 .

We show the existence of an implied subsidy of capital in section 6 for the finite horizon case. There, we also discuss its economic interpretation. In the infinite horizon case, we can demonstrate even stronger predictions: all economic agents will eventually have a non-positive intertemporal wedge (see section 7 ). 


\section{Output and consumption distribution}

We start our analysis of the general setting with the allocation of consumption and output across agents. First we want to know whether agents allocate between consumption and leisure optimally or incentive provision instead dictates that the marginal rate of substitution and transformation shall not be equated. In particular, we explore how allocations compare between an agent whose skill level becomes public information and an agent with identical history and skill level but the latter remains private information.

The derivations of the first-order necessary conditions can be found in the Appendix. There, we also prove that constrained efficient allocations are interior, which we use to simplify our presentation whenever possible. ${ }^{8}$

Let us first consider the case when the agent's skill level remains private information, $a_{t}=0$. We find that the results of the classic, static Mirrlees economy carry over. An agent with skill $\theta_{t}$ will face a wedge between the marginal rate of substitution and the marginal rate of transformation as long as some other agent $\theta^{\prime}$ incentive-compatibility constraint to report the type $\theta_{t}$ is binding, that is, the Lagrangian multiplier $\mu\left(s^{t-1}, \theta^{\prime} ; \theta_{t}\right)$ is positive. By encouraging leisure the allocation prescribed for skill level $\theta_{t}$ looks less attractive to agents with higher skill levels. Again as in Mirrlees (1971), no agent has incentives to report the top skill level, and thus the marginal rate of substitution and the marginal rate of transformation are equated for agents with top skill. Note that the presence of positive marginal labor taxes is assured as long as private information constrains the set of admissible allocations. ${ }^{9}$ We summarize these findings in the following proposition.

\section{Proposition 1 (Mirrleesian results)}

For any date $t=0,1, \ldots, T$ and node $s^{t-1} \in S^{t-1}$ with $a_{t}=0$,

$$
h^{y}\left(s^{t} ; \theta_{t}\right) \leq u^{\prime}\left(s^{t}\right) F_{t}^{y}
$$

with strict inequality iff $\mu\left(s^{t-1}, \theta^{\prime} ; \theta_{t}\right)>0$ for some $\theta^{\prime} \in \Theta$.

Proof: In the Appendix.

We now turn our attention to allocation whenever the skill level becomes public information, $a_{t}=1$. Obviously, allocations conditional on $a_{t}=1$ are not part of the payoff of misreporting for any agent. It is thus possible to relax the incentive-compatibility constraints by prescribing different allocations in the event $a_{t}=1$. Indeed we find that the constrained efficient allocation

\footnotetext{
${ }^{8}$ See Proposition 6 .

${ }^{9}$ Our assumptions on preferences rule out the possibility of "bunching," in which some agents with skill level $\theta_{t}<\bar{\theta}$ face a zero marginal tax. See Tuomala (1990) and Salanie (2003) for a discussion and references herein.
} 
does so.

First, there is no need to distort the labor supply decision of agents whose type is public information. The only reason for the labor supply to be distorted for some agents is to ease the incentives to misreport. But there is no need to do so for the allocation with observable skills. In this case, delivering the efficient allocation between consumption and leisure relaxes the incentive-compatibility constraints (4). Any tax system that would decentralize the constrained efficient allocation would need to include a provision for a zero marginal tax rate - a tax breakwhenever the skill level of an agent is public information.

We go one step further by ranking consumption and labor allocations between agents with and without state verification. We find that for two agents with identical histories $s^{t-1}$ and identical skill level $\theta_{t}$, consumption is weakly higher for the agent whose type is public information,

$$
c\left(\left\{s^{t-1}, \theta_{t}, 0\right\}\right) \leq c\left(\left\{s^{t-1}, \theta_{t}, 1\right\}\right)
$$

with strict inequality as long as the incentive-compatibility constraint is binding. The possibility of state-verification thus introduces consumption variation along the equilibrium path. Similarly the agent with observable skills enjoys not only more consumption but also more leisure:

$$
y\left(\left\{s^{t-1}, \theta_{t}, 0\right\}\right) \geq y\left(\left\{s^{t-1}, \theta_{t}, 1\right\}\right) .
$$

Furthermore, the agent with observable skills will also continue to receive higher utility than the agent. We infer this observation from a higher Lagrange multiplier on the constraint on promised utility.

Agents are thus "rewarded" whenever their skill level becomes public information by having more consumption and more leisure, as well as more continuation welfare. By delivering higher welfare in the event of state verification, the agent's incentive-compatibility constraints are relaxed without tightening any other agent's constraints. The variation in consumption, though, is costly since agents are risk averse. Risk aversion thus puts a bound on the optimal spread in consumption levels. If agents were risk neutral, consumption and welfare would exhibit a bang-bang solution.

Proposition 2 formalizes these results.

\section{Proposition 2 (Rewards for state verification)}

For any date $t=0,1, \ldots, T$ and node $s^{t-1} \in S^{t-1}$,

$$
\begin{array}{r}
c\left(\left\{s^{t-1}, \theta_{t}, 0\right\}\right) \leq c\left(\left\{s^{t-1}, \theta_{t}, 1\right\}\right) \\
y\left(\left\{s^{t-1}, \theta_{t}, 0\right\}\right) \geq y\left(\left\{s^{t-1}, \theta_{t}, 1\right\}\right)
\end{array}
$$


for all $\theta_{t} \in \Theta$, and with strict inequality iff $\mu\left(s^{t-1}, \theta^{\prime} ; \theta_{t}\right)>0$ for some $\theta^{\prime} \in \Theta$. Moreover, if $a_{t}=1$,

$$
h^{y}\left(s^{t} ; \theta_{t}\right)=u^{\prime}\left(s^{t}\right) F_{t}^{y} .
$$

Proof: In the Appendix.

Finally, we turn our attention to the agent with the highest skill level $\bar{\theta}=\max \{\theta \in \Theta\}$. Since our preferences satisfy the single-crossing condition, the incentive-compatibility constraints for agents with $\theta_{t}<\bar{\theta}$ to report $\bar{\theta}$ are not binding. It follows from Proposition 2 that the allocations of consumption, output, and promised utiilty for the agent with top skill level are identical whether the skill level is made public information or remains private information. The reason is that no agent with $\theta_{t}<\bar{\theta}$ has incentives to misreport their type as $\bar{\theta}$. Thus prescribing distinct allocations for $a_{t}=0$ and $a_{t}=1$ does not relax any incentive-compatibility constraint. Since it does not improve incentives, providing the same allocation whether the state is verified or not delivers the same welfare to the top skill agent with less resources.

\section{The incentives to save}

This section is concerned with the allocation of resources across periods. This topic has been the focus of most of the literature on dynamic Mirrleesian economies, starting with the finding in Golosov, Kocherlakota and Tsyvinski (2003) that the marginal benefit of investment is above the marginal cost. The standard dynamic Mirrleesian economy is nested in our setup when the probability of state verification equals zero.

Since aggregate capital does not directly affect incentives, the first-order condition for $K_{t}$ equates the shadow cost of resources $\lambda_{t}$ across periods as the efficient allocation would do. Of course, the shadow cost of resources will generally not be the same for efficient and constrainedefficient allocations, and thus the optimal paths for aggregate capital will differ as well. Without further assumptions it is difficult to say much about the transition path for capital.

Fortunately we can characterize the incentives to save for private agents. Proposition 6 in the Appendix shows that consumption and output are strictly positive in the constrained efficient allocation so the first-order conditions hold with strict equality. Given this result, we follow the same steps involved in the derivation of the inverse-Euler equation - see Rogerson (1985) and Golosov, Kocherlakota and Tsyvinski (2003). The resulting optimality condition for intertemporal allocations reads

$$
u^{\prime}\left(s^{t}\right)=\left(E_{t}\left\{\frac{1}{u^{\prime}\left(s^{t+1}\right)}\right\}\right)^{-1}\left(1+p \frac{\mu^{*}\left(s^{t}\right)}{1+\eta\left(s^{t}\right)}\right)
$$


where

$$
\mu^{*}\left(s^{t}\right)=\sum_{\theta, \theta^{\prime} \in \Theta}\left\{\pi(\theta) \mu\left(s^{t}, \theta ; \theta^{\prime}\right)\right\}
$$

is a weighted average of Lagrange multipliers $\mu\left(s^{t}, \theta ; \theta^{\prime}\right)$ on the incentive compatibility constraints.

If there is no chance that the state is verified, $p=0$, we recover the familiar inverse Euler equation from (8). Following Golosov, Kocherlakota and Tsyvinski (2003), we can establish the existence of a positive wedge between the marginal benefit and the marginal cost of investment, as

$$
u^{\prime}\left(s^{t}\right)=\left(E_{t}\left\{\frac{1}{u^{\prime}\left(s^{t+1}\right)}\right\}\right)^{-1}<E_{t}\left\{u^{\prime}\left(s^{t+1}\right)\right\}
$$

where the last step follows from Jensen's inequality. If we were to decentralize the constrained efficient allocation in the case $p=0$, we would find that we need a positive capital tax. In short, reducing savings helps in improving the provision of incentives in future periods, as it becomes easier to provide the necessary spread in the marginal utility of consumption.

On the other end of the spectrum where all the skill types are observed, information is no longer private. Hence, the first-best allocation becomes feasible for the planner. The interesting results of this paper thus deal with probabilities in between these two extrema.

Once we consider the possibility of state-verification, though, we can no longer establish unambiguously that the marginal benefit of investment is above the marginal cost, or vice versa. The sign of the intertemporal wedge $u^{\prime}\left(s^{t}\right)-E_{t}\left\{u^{\prime}\left(s^{t+1}\right)\right\}$ depends on the size of Lagrange multipliers and probabilities of state verification. ${ }^{10}$

Random state-verification opens a new channel to improve incentives for future periods. Recall that an agent caught misreporting her type is assigned continuation welfare equal to $\Upsilon$. Thus the amount of welfare forgone once caught misreporting is $z\left(s^{t}\right)-\Upsilon$, what we call the effective punishment. Incentive provision is better the harsher is the effective punishment. Everything else equal, the planner can improve allocations by promising higher continuation welfare. In turn, higher continuation welfare requires accumulating more capital in order to generate the necessary additional resources.

However, the opposite force of standard private information economies remains: incentives require a larger consumption spread when there are more savings. Whether the constrained efficient allocation prescribes a positive or negative intertemporal wedge (8) depends on which channel is stronger. In other words, savings present the planner with a trade-off between more effective punishment and a larger consumption spread.

\footnotetext{
${ }^{10}$ The exception is when $\mu^{*}=0$. In this case no incentive-compatibility constraint is binding at nodes $s^{t+1} \in$ $S^{t+1} \mid s^{t}$, and the allocation features full insurance with respect to date $t+1$ shocks.
} 
The random-state verification channel has a key property: the effective punishment can be large enough to overcome all the incentive problems. That is, if the continuation welfare is high enough, then no agent risks losing it by misreporting, even if the allocations prescribe full insurance and no labor distortions. For constrained-efficient allocations we can say even more: full insurance and no labor distortions are necessary properties if the continuation welfare is above a certain threshold. Proposition 3 formalizes the first key result of the paper.

\section{Proposition 3}

Let $p>0$. There exists threshold $z^{*}<\infty$ such that if $\{x, K, z\}$ is a constrained efficient allocation and

$$
z\left(s^{t-1}\right) \geq z^{*}
$$

then for all dates $j \geq t$ and continuation nodes $s^{j} \in S^{j} \mid s^{t-1}$ there are no labor distortions:

$$
v^{\prime}\left(y\left(s^{j}\right) / \theta_{j}\right)=u^{\prime}\left(c\left(s^{j}\right)\right) \theta_{j}
$$

and there is full insurance:

$$
c\left(s^{j}\right)=c\left(s^{t}\right)
$$

for all $s^{t} \in S^{t} \mid s^{t-1}$.

Proof: In the Appendix.

The proof of proposition 3 is quite straightforward. The welfare gains for misreporting the skill type and going undetected are bounded above when the efficient allocations are prescribed. We thus only need a large enough effective punishment to enforce truthful reporting.

Proposition 3 does not imply that the upper bound will ever be reached. Whether we will reach the range of full insurance is a matter of discussion in the following sections. However, it already tells us that we cannot have very high levels of promised utility and still have the same prediction of a positive intertemporal wedge as for standard dynamic Mirrleesian economies.

We highlight next a key implication of Proposition 3 that is featured prominently in our results for finite and infinite horizon economies.

\section{Proposition 4}

Let $p>0$ and $\{x, K\}$ be a constrained efficient allocation. If $\{x, K\}$ is not efficient, then

$$
c\left(s^{t}\right) \leq \bar{c}<\infty
$$

for all nodes $s^{t} \in S^{t}$ and dates $t=0,1, \ldots, T$.

Proof: In the Appendix. 
Note this stands in contrast with the standard Mirrleesian economy with $p=0$ : individual consumption is usually unbounded above.

Proposition 3 states that the constrained-efficient allocation will have some surprising properties if the continuation welfare is large enough. However, we still do not know whether it is part of the constrained-efficient allocation to back-load welfare until the threshold in Proposition 3 is achieved. Doing so welfare is not costless: it requires low initial consumption, and in general a less smooth path for consumption. In the next sections we will characterize the conditions under which the random state-verification channel dominates and the marginal return to investment is below its cost in constrained efficient allocations.

\section{Finite Horizon}

In this section, we turn to finite horizon economies with an arbitrary time horizon. We already know the results for a special case. Section 3 discussed a two period economy. In that section, we saw the size of intra- and intertemporal wedges for a particular calibration.

Besides the special case, we gathered a fair bit of information on the finite horizon economy. We know that the distortions for the labor supply decision has the same qualitative properties as in a standard dynamic Mirrleesian economy, as section 4 shows. There, we also discussed the ranking of consumption and output allocations. Section 5 discussed the optimality condition for the intertemporal allocation. Compared to the standard Mirrleesian setting, the intertemporal wedge has an extra term that prevents us from determining the sign of the wedge directly.

This section sheds more light on the question in which cases the intertemporal wedge is negative or positive. Therefore, we discuss two sets of results. First, for any finite horizon economy, the implied savings tax is negative if the probability of state verification is in a certain range. And second, for a given (potentially tiny) probability of state verification, there exists a time horizon $T$ such that the implied savings tax is negative for some agents in the economy.

Let us turn to the first result. As we have seen in previous sections, a higher probability of state verification relaxes the incentive compatibility constraints. We can get intuition by studying two extreme cases. On one end of the spectrum, no skill level gets observed. In this case, we retrieved the standard dynamic Mirrleesian setup for which we know that the intertemporal wedge is positive and hence, the implicit tax on savings is positive. On the other end of the spectrum, all skill levels become public information before allocations get assigned. Since there is no private information, no incentive compatibility constraints have to be satisfied and we recover the first best allocation in which full risk sharing prevails.

For the two period economy, however, we showed that the intertemporal wedge does not 
decline monotonically. It rather turns into a subsidy region before it enters the range in which the first best allocation can be delivered. The rationale for the subsidy region is given by the fact that for a higher probability of state verification, more savings relax incentive constraints for two reasons. First, the effective punishment in case of misreporting increases. But second, with higher probabilities of the skill becoming public information, every extra unit of effective punishment has a bigger effect on the expected punishment.

We now show that the existence of a subsidy is a general result that holds for any finite horizon economy of our setup. We therefore prove the following proposition.

\section{Proposition 5}

For any $T$ and node $s^{t}, t<T$ there exists $p<p^{*}$ such that

$$
u^{\prime}\left(s^{t}\right)<E_{t}\left\{u^{\prime}\left(s^{t+1}\right)\right\}
$$

\section{Proof: TBC}

The key to this result lies in the incentive compatibility constraints. Imagine an economy with the lowest probability of state verficiation that still supports the first-best outcome. The fact that the full consumption sharing can be implemented means that the effect of private information has been overcome. In essence, the effective punishment is strong enough to allow full risk sharing. That means, however, that the reason for front-loading also has been eliminated. If the probability of state verification is however just a slight bit smaller, the effective punishment channel calls for a subsidy to push agents towards the region of a first-best allocation. Since the effect of front-loading consumption is marginal, the intertemporal wedge becomes negative.

But is a subsidy an option for any given probability of state verification? Surprisingly, the answer is yes - provided that the time horizon is long enough. The next theorem shows that any economy with positive probability of skills becoming observable features a subsidy.

\section{Theorem 1}

Let $p>0$. There exists $T<\infty$ such that the constrained efficient allocation $\{x, K\}$ satisfies

$$
u^{\prime}\left(s^{t}\right) \geq E_{t}\left\{u^{\prime}\left(s^{t+1}\right)\right\}
$$

for some node $s^{t} \in S^{t}, t \leq T-1$.

Proof: In the Appendix

Intuitively, we know that for a given probability $p$, there is a level of savings such that you can give agents first best allocations. Now the question remains of whether agents will ever reach that level. Therefore, consider the relevant trade-offs in a given period. To induce agents 
to work, they need to be given consumption in return. However, this consumption does not have to be delivered in the current period. It can merely be promised to be delivered in future periods. The promise keeping constraint ensures that eventually consumption will be provided. But therefore, the economy has to build up enough savings which in turn raises the effective punishment level. If the time horizon is long enough, a sufficient amount of savings guarantees that some agents have a non-positive intertemporal wedge.

Theorem 1 raises the question of what happens if the time horizon is infinite. The next section shows a surprising result: not only sum but all agents (but a set of zero mass) will reach the non-positive intertemporal wedge.

\section{$7 \quad$ Infinite horizon economies}

This section answers which sign the intertemporal wedge takes on in infinite horizon economies. We find that not only do some but all agents (but a set of mass zero) converge to either a negative intertemporal wedge or a first-best allocation. Even more interestingly, this result does

not depend on the probability of state verification. Even a tiny chance of skill levels being revealed leads agents to converge to a non-positive intertemporal wedge.

Let us be clear about the precise meaning of the concept of convergence. Our results on the long-run are stated in terms of convergence in probability. ${ }^{11}$ For example, we will say that consumption converges to some level $\hat{c}$, denoted $c\left(s^{t}\right) \rightarrow \hat{c}$, if for any $\delta>0$

$$
\lim _{t \rightarrow \infty} \sum_{s^{t} \in S^{t}} \pi\left(s^{t}\right) \chi\left[\left|c\left(s^{t}\right)-\hat{c}\right| \geq \delta\right]=0
$$

where $\chi$ is the indicator function. The long-run properties will thus hold for all agents with the possible exception of a subset with measure zero.

The following theorem summarizes the main result of this section.

\section{Theorem 2}

Let $p>0$. The economy converges to full insurance and no labor distortions:

$$
c\left(s^{t}\right)=c\left(s^{t+1}\right)
$$

and

$$
v^{\prime}\left(y\left(s^{t}\right) / \theta_{t}\right)=u^{\prime}\left(c\left(s^{t}\right)\right) \theta_{t}
$$

for all $\theta_{t} \in \Theta$.

\footnotetext{
${ }^{11}$ See Stokey and Lucas (1996) for a formal treatment.
} 
Proof: In the Appendix

The rest of this section is concerned with shedding some more light on this insight. Therefore, we first establish existence of an absorbing upper bound on promised welfare. Then we show that there is a non-absorbing lower bound which means that agents close to that bound can reach higher promised welfare after good shock realizations. To establish the result of the theorem, the remaining piece is to show that the Lagrange multiplier on the promised welfare constraint follows a sub-martingale. We apply the sub-martingale convergence theorem to prove the result of theorem 2. Note that the Lagrangian multiplier associated with the resource constraint (2) is constant due to constant marginal returns to capital. This assumption becomes important in the infinite horizon case as it allows the economy to convergence to an arbitrarily large level of aggregate capital and thus defer the delivery of utility to the future.

As demonstrated in proposition 3, there exists an upper threshold on promised utility such that full insurance without labor distortions can be implemented. The intuition for this upper bound goes as follows. The larger the punishment associated with misreporting, the more can the state-verification technology relax the incentive-compatibility constraints. The continuation welfare prescribed in the event of misreporting is exogenously fixed at $\Upsilon$. However, the right measure of punishment is the amount of "forgone" welfare, that is, the difference between the promised continuation welfare and the punishment. There is a level of continuation welfare $z^{*}$ for which the threat of punishment is so large that the effects of private infomration are dominated and full insurance can be implemented.

Our next result goes one step further and shows that full insurance with no labor distortions is a necessary property of the constrained efficient allocation whenever promised utility is at or above the threshold for full insurance. Intuitively, for any level of promised utility, the costefficient solution to the promise-keeping constraint is precisely to provide full insurance with no labor distortions. Under the conditions in proposition 3, the incentive-compatibility constraints are no longer an impediment to do so.

As can be seen in the following corollary, proposition 3 implies that $z\left(s^{t}\right) \geq z^{*}$ is an absorbing state, as the promised continuation welfare will be constant under full insurance.

\section{Corollary 1}

Let $p>0$. If $z\left(s^{t}\right) \geq z^{*}$ then $z\left(s^{j}\right)=z\left(s^{t}\right)$ for all $\left\{s^{j}: s^{t} \in s^{j}, j \geq t\right\}$.

So far we have established that there exists an upper bound on continuation welfare, and that it is absorbing state. We now turn to showing that there is also a non-absorbing lower bound.

By assumption there is a lower bound on welfare (1). We briefly discuss how continuation welfare behaves close to the lower bound. We find that the lower bound on continuation welfare 
(1) may be binding with positive probability. However, promised continuation welfare is always strictly higher than the lower bound.

\section{Lemma 2}

Let $\{x, K\}$ be a constrained efficient allocation and $z$ the associated plan of continuation welfare. Then $z\left(s^{t}\right)>\Upsilon$.

Proof: In the Appendix

The key implication of Lemma 2 is that the lower bound is not absorbing. Instead an agent hitting the lower bound suffers a very low per-period welfare but is promised continuation welfare above the lower bound. ${ }^{12}$

We now link the results above with the stochastic process for the multiplier $\eta\left(s^{t}\right)$ associated with the promise-keeping constraint (3). The multiplier effectively governs the process for continuation-welfare. An upper bound $\eta^{*}$ can be derived from proposition 3, and characterizes an absorbing state. There is a lower bound as well that is transient as shown in Lemma 2 .

The key equation is the characterization of the one-step ahead expectation which is given by

$$
E_{t}\left\{\eta\left(s^{t+1}\right)\right\}=\eta\left(s^{t}\right)+p \mu^{*}\left(s^{t}\right) .
$$

As long as $p>0$ and some incentive-compatibility constraint is binding, the multiplier $\eta\left(s^{t}\right)$ follows a submartingale process with a strictly positive drift. The process is bounded above and below as established by proposition 3 and Lemma 2. There is an absorbing state, as established by Corollary 1. These conditions allow us to apply the sub-martingale convergence theorem to show that the multiplier $\eta\left(s^{t}\right)$ converges to the upper bound, and so does continuation welfare. Hence, in the long run, the economy features full insurance and no labor distortion.

Distortions introduced by private information are thus temporary in the sense that they vanish in the long run for all agents but a subset of measure zero. We saw in proposition 3 that the economy can "outgrow" the incentive-compatibility constraints by promising higher continuation welfare. However to sustain the welfare promises it is necessary to accumulate aggregate capital and this transition is costly.

Why then does the economy converge to full insurance independent of initial conditions, discount rate, and state-verification probability? The key insight is that, under state-verification, back-loading welfare can help to ease incentives. If we need to provide incentives to a high-skill agent at date $t$ to reveal her type, we can increase consumption allocated to high types at date $t$; or we can increase the promised continuation welfare from date $t+1$ onwards. The latter is more efficient because it also improves the incentives at date $t+1$ by increasing the amount of

\footnotetext{
${ }^{12}$ Our results closely mirror those in Atkeson and Lucas (1993).
} 
welfare forgone in case of being caught misreporting. Yet, there are othere forces working in the opposite direction. First, concavity favors smooth consumption paths. Second, the rationale for front-loading welfare in the standard private information economies remains: to deliver higher promised welfare at date $t+1$ the planner must prescribe a larger consumption spread between skill levels to ensure that the high skill type truthfully reveals her type.

As promised utility becomes larger, the punishment channel becomes more effective and the private information channel becomes weaker because the dispersion of consumption will be lower. Hence, for very high promised utilities, there is essentially no trade-off.

Hence, in the long-run, the incentives for back-loading welfare eventually dominate. The key feature of an infinite-horizon economy is that every agent receives a series of high-skill realizations of arbitrary (but finite) length with probability one. During this "streak" promised welfare is naturally increasing and eventually the state-verification effect dominates.

The result in Theorem 2 stands in stark contrast with the results in dynamic Mirrleesian economies without state-verification. In these economies, aggregate consumption is decreasing. No agent ever achieves full insurance and labor distortions are always present. It may seem puzzling that even an arbitrarily small state-verification probability can lead to such different results. However, these are long-run properties: the welfare improvement at any date associated with a small state-verification probability may be small.

\section{Extensions}

This section discusses three possible extensions to our model and their implications for the results. Throughout the paper, we assumed a production function with constant marginal returns to capital. The subsection thus discusses how the implementation of a production function with decreasing marginal returns to capital would alter our predictions. We then turn to endogenizing the punishment level. In the last subsection, we discuss an endogenous choice of state verification.

\subsection{Decreasing marginal returns to capital}

We characterized the constrained-efficient allocations for a linear production function. However, our results concerning the consumption and output distribution, as well as the finite horizon properties, apply to more general constant-returns-to-scale production functions.

The assumption of constant marginal returns to capital plays a key role in the long-run results. Theorem 2 establishes convergence to full insurance in the long run. For full insurance to be incentive compatible, we need the level of consumption to be high enough for the punishment 
to be effective. Thus, in the long run, the economy must be able to accumulate enough capital to sustain a large level of aggregate consumption. In economies with decreasing marginal returns to capital, this may not be feasible.

Consider a more general constant returns to scale production function and let $f\left(K_{t}\right)$ be output per effective unit of labor net of capital depreciation. Decreasing marginal returns to capital imply that $f$ is a strictly concave function. Steady-state aggregate consumption is maximized at capital level $K_{t}=\bar{K}$ with $f^{\prime}(\bar{K})=1$. Thus aggregate consumption is bounded above. We conjecture that, if $\bar{K}$ is high enough, Theorem 2 would still apply.

It is harder to venture a conjecture for the long-run properties in the case where the upper bound $\bar{K}$ is not high enough to sustain full insurance. In particular, we did not find any guarantee that there exists a stationary consumption distribution. No agent will enjoy full insurance for all continuing dates with probability one. However, there may be a subset of agents enjoying full or almost full insurance at any given date. This suggests that, if there exists a stationary distribution, it will be skewed to the left, with low consumption households facing higher consumption volatility than households with high average consumption.

\subsection{Endogenous punishment level}

In our setup, incentives improve with stronger punishments. We took the punishment level as exogenous to focus on the implications of random state verification. Here we briefly discuss possible theories on potential levels of punishment.

One possibility is that an agent caught misreporting her type is prevented from all trading and savings, as in the limited commitment models of Kehoe and Levine (1993) and Kocherlakota (1996). The punishment level for an agent with type $s^{t}$ is then given by

$$
\Upsilon\left(s^{t}\right)=\max _{y\left(s^{j}\right): s^{j} \in S^{j} \mid s^{t}} \sum_{j=t}^{\infty} \sum_{\theta_{j} \in \Theta} \beta^{j-t} \pi\left(\theta_{j} \mid \theta_{t}\right)\left(u\left(y\left(s^{j}\right)\right)-v\left(y\left(s^{j}\right) / \theta_{j}\right)\right) .
$$

The punishment level given by autarky does not depend on the full history: only the present realization of skill level $\theta_{t}$ matters. Importantly, none of the allocations prescribed on the equilibrium path change the value of punishment. In this case the first order conditions are unchanged, and all of our results remain valid.

Essential to our results concerning the intertemporal wedge is the ability of the planner to strengthen the effective punishment by increasing continuation welfare. If the punishment level scales up with continuation welfare, our results may change. For example, if we prescribe autarky as punishment but we allow the agent to hold on to her savings, then the effective

punishment may decrease with savings, reversing our arguments. Similarly, if the punishment 
is modeled a fixed drop in utility, the effective punishment becomes constant. That said, we are confident that a satisfactory theory of punishment would allow for the effective punishment to increase with continuation welfare.

\subsection{Towards a theory of optimal monitoring}

Perhaps the most unsatisfactory aspect of our analysis is that state verification is a random event, out of the planner's control. We would like to allow the planner to specify a different state verification probability for agents with different types or reports, and perhaps at different dates.

We can extend the model to allow for type-specific, or report-specific state verification probabilities. Doing so can bring the random state verification technology closer to an optimal monitoring strategy. For example, it is clear that there is no point in verifying the state of an agent reporting to have high skill, as no agent with low skill will find it beneficial to misreport a higher skill level. Thus we can set this particular state verification probability to zero. Our results clearly depend only on the probability showing up in binding incentive-compatibility constraints; as long as these are positive we expect our analysis to be valid.

However, in a fully optimal monitoring schedule, state verification probabilities would be history dependent. The planner is likely to focus its state verification efforts where incentives are the worst. There will not be much use to monitor heavily agents with a high continuation welfare, i.e., with a large effective punishment. This form of history-dependence, though, complicates the analysis greatly.

\section{Conclusions}

We set out to take a first step towards a more realistic information framework for dynamic Mirrleesian economies. From observing the implementation of social insurance schemes and the prevention of fraud, we introduced an exogenous state-verification technology. The skill level of any agent becomes public information with some given probability. The planner can specify allocations as function of the reported skill level, whether the skill level is observable and, if so, the actual skill level.

Our results establish that state-verification can radically shape the constrained efficient allocation. In particular in the infinite horizon economy with constant returns to capital, we discover very strong predictions. We find that even an arbitrarily small probability of stateverification implies that the economy converges to full insurance and all labor distortions vanish in the long run. In finite horizon economies we show that different agents at different times will 
generally have different incentives-to-save, and a decentralization would require that savings are sometimes taxed and sometimes subsidized.

A potential shortcoming of our analysis is that we may be missing some key features of a properly designed audit mechanism by taking the state-verification technology to be exogenous. First, we assumed that the probability of verifying an agent's skill level is independent of the message. There is, however, no need to audit agents who report to be of high skill type as no one would have the incentive to pretend to have the highest skill. Second, the lower bound on welfare may reflect some feasibility restriction, an outside option for agents, or some limitation in the government's ability to commit to stern punishments. Finally, we have abstracted from the costs associated with an audit. It turns out that our specification of the state-verification can address most of the issues above. It is possible to work with skill-specific state-verification probabilities and our results remain unchanged. Since the revelation principle applies, this is identical to having an audit probability conditional on the report. An endogenous specification of the punishment level would be, of course, more satisfactory although off-equilibrium events remain elusive by their very nature.

With respect to the costs of an audit, there is no difficulty to model a resource cost associated with state-verification. The challenge, though, is to incorporate some non-transferrable costs. For example, the literature on imperfect monitoring suggests that a noisy signal for the skill level leads to bang-bang strategies: the punishment will be implemented with positive probability in this environment even if all agents are known to report truthfully. 


\section{A Proofs for Section 4}

We repeat the optimal control problem of the main text. We maximize

$$
\sup _{\{x, K, z\}} W_{0}(x)
$$

subject to the incentive-compatibility and promise-keeping constraints (4) and (3) at all nodes $s^{t} \in S^{t}$, and the resource constraint (2) at all dates $t=0,1, \ldots, T$, as well as non-negativity constraints and the lower bound constraint on continuation welfare (1).

By Assumption 1, $h\left(x\left(s^{t}\right) ; \theta_{t}\right)$ is twice differentiable in $\Re_{+}^{2}$ for all $\theta_{t} \in \Theta$. We can thus characterize the constrained efficient allocation with the Lagrangian

$$
\begin{aligned}
& \mathscr{L}(x, K, z ; \lambda, \eta, \mu)=W_{0}(x)-\sum_{t=0}^{T} \beta^{t} \lambda_{t}\left\{C_{t}+K_{t+1}-F\left(K_{t}, Y_{t}\right)\right\} \\
& -\sum_{t=0}^{T-1} \sum_{s^{t} \in S^{t}} \beta^{t} \pi\left(s^{t}\right) \eta\left(s^{t}\right)\left\{z\left(s^{t}\right)-\sum_{s^{t+1} \in S^{t+1}} \pi\left(s^{t+1} \mid s^{t}\right)\left\{h\left(x\left(s^{t+1}\right) ; \theta_{t+1}\right)+\beta z\left(s^{t+1}\right)\right\}\right\} \\
& -\sum_{t=0}^{T} \sum_{s^{t} \in S^{t}} \beta^{t} \pi\left(s^{t}\right) \zeta\left(s^{t}\right)\left\{\left(1-\beta^{T+1-t}\right) \Upsilon-h\left(x\left(s^{t}\right) ; \theta_{t}\right)-\beta z\left(s^{t}\right)\right\} \\
& -\sum_{t=0}^{T} \sum_{s^{t-1} \in S^{t-1}} \sum_{\theta_{t}, \theta^{\prime} \in \Theta} \beta^{t} \pi\left(s^{t-1}, \theta_{t}\right) \mu\left(s^{t-1}, \theta_{t} ; \theta^{\prime}\right) \\
& \left\{\sum_{a_{t} \in\{0,1\}} \pi\left(a_{t}\right)\left\{h\left(x\left(s^{t}\right) ; \theta_{t}\right)+\beta z\left(s^{t}\right)\right\}-p\left(1-\beta^{T+1-t}\right) \Upsilon-(1-p)\left\{h\left(x\left(\hat{s}^{t}\right) ; \theta^{\prime}\right)+\beta z\left(\hat{s}^{t}\right)\right\}\right\} .
\end{aligned}
$$

We have omitted from the Lagrangian the non-negativity constraints on consumption and output as well as the terminal condition on $z\left(s^{T}\right)$ for finite horizon economies.

Next we state the necessary first order conditions. For nodes $s^{t}$ when the state remain private information, $a_{t}=0$, we have:

$$
\begin{gathered}
u^{\prime}\left(s^{t}\right)\left(1+\eta\left(s^{t-1}\right)+\sum_{\theta^{\prime} \in \Theta}\left\{\mu\left(s^{t-1}, \theta_{t} ; \theta^{\prime}\right)-\frac{\pi\left(\theta^{\prime}\right)}{\pi\left(\theta_{t}\right)} \mu\left(s^{t-1}, \theta^{\prime} ; \theta_{t}\right)\right\}\right) \leq \lambda_{t}, \\
h^{y}\left(s^{t} ; \theta_{t}\right)\left(1+\eta\left(s^{t-1}\right)+\sum_{\theta^{\prime} \in \Theta}\left\{\mu\left(s^{t-1}, \theta_{t} ; \theta^{\prime}\right)-\frac{\pi\left(\theta^{\prime}\right)}{\pi\left(\theta_{t}\right)} \mu\left(s^{t-1}, \theta^{\prime} ; \theta_{t}\right) \frac{h^{y}\left(s^{t} ; \theta^{\prime}\right)}{h^{y}\left(s^{t} ; \theta_{t}\right)}\right\}\right) \geq \lambda_{t}, \\
\eta\left(s^{t-1}\right)+\sum_{\theta^{\prime} \in \Theta}\left\{\mu\left(s^{t-1}, \theta_{t} ; \theta^{\prime}\right)-\frac{\pi\left(\theta^{\prime}\right)}{\pi\left(\theta_{t}\right)} \mu\left(s^{t-1}, \theta^{\prime} ; \theta_{t}\right)\right\} \leq \eta\left(s^{t}\right) .
\end{gathered}
$$


For nodes when the skill level is observable, $a_{t}=1$, we have:

$$
\begin{aligned}
u^{\prime}\left(s^{t}\right)\left(1+\eta\left(s^{t-1}\right)+\sum_{\theta^{\prime} \in \Theta} \mu\left(s^{t-1}, \theta_{t} ; \theta^{\prime}\right)\right) & \leq \lambda_{t}, \\
h^{y}\left(s^{t} ; \theta_{t}\right)\left(1+\eta\left(s^{t-1}\right)+\sum_{\theta^{\prime} \in \Theta} \mu\left(s^{t-1}, \theta_{t} ; \theta^{\prime}\right)\right) & \geq \lambda_{t}, \\
\eta\left(s^{t-1}\right)+\sum_{\theta^{\prime} \in \Theta} \mu\left(s^{t-1}, \theta_{t} ; \theta^{\prime}\right) & \leq \eta\left(s^{t}\right) .
\end{aligned}
$$

In addition f.o.n.c. on aggregate capital is:

$$
\lambda_{t}=\lambda_{t+1} \beta R,
$$

for all dates $t<T$. Given our assumption that $\beta R=1$, we have that $\lambda_{t}=\lambda_{t+1}$ for all dates $t<T$.

The next Proposition guarantees that the constrained efficient allocation is interior at all nodes.

\section{Proposition 6}

Let $\{x, K\}$ be a constrained efficient allocation. Then for all $s^{t} \in S^{t}, t=0,1, \ldots, T$

$$
\begin{array}{r}
c\left(s^{t}\right) \geq c^{b}>0 \\
y\left(s^{t}\right)>0
\end{array}
$$

Proof: By (1) any admissible allocation must satisfy

$$
u\left(c\left(s^{t}\right)\right) \geq u\left(c\left(s^{t}\right)\right)-v\left(\frac{y\left(s^{t}\right)}{\theta_{t}}\right) \geq v .
$$

Assumptions 1 and 3 guarantees there exists $c^{b}>0$ that achieves this lower bound, and thus for all admissible allocations $c\left(s^{t}\right) \geq c^{b}>0$. Next we show $y\left(s^{t}\right)>0$ if $\{x, K\}$ is a constrained efficient allocation. Assume $y\left(s^{t}\right)=0$ for any node. By Assumption $1, v^{\prime}(0)=0$. The necessary first order conditions associated with the Lagrangian imply then $0 \geq \lambda_{t} F_{t}^{y}$, and thus we reach a contradiction.

Proof: [Proof of Proposition 1] Our preferences satisfy the single-crossing condition and 
thus $\mu\left(s^{t-1}, \theta^{\prime} ; \theta_{t}\right)=0$ for $\theta^{\prime}<\theta_{t}$. Using the strict convexity of $h$,

$$
\mu\left(s^{t-1}, \theta^{\prime} ; \theta_{t}\right) h^{y}\left(s^{t} ; \theta^{\prime}\right) \leq \mu\left(s^{t-1}, \theta^{\prime} ; \theta_{t}\right) h^{y}\left(s^{t} ; \theta_{t}\right)
$$

Combining (9)-(10) and using the inequality above we obtain the result.

Proof: [Proof of Proposition 2] By Proposition 6 the system of f.o.n.c. (9)-(10) and (12)-(13) holds with strict equality. Comparing (9) and (12) it is clear that

$$
u^{\prime}\left(s^{t-1}, \theta_{t}, 0\right) \geq u^{\prime}\left(s^{t-1}, \theta_{t}, 1\right)
$$

with strict equality iff $\mu\left(s^{t-1}, \theta^{\prime} ; \theta_{t}\right)=0$ for all $\theta^{\prime} \in \Theta$. Since $u$ is strictly concave, the result on consumption follows. A similar argument applies for output, now comparing (10) and (13). To show that $h^{y}\left(s^{t} ; \theta_{t}\right)=u^{\prime}\left(s^{t}\right) F_{t}^{y}$ in case $a_{t}=1$ combine (12)-(13).

\section{B Proofs for Section 5}

\section{Proof: [Proof of Proposition 3]}

The proof proceeds as follows. First we construct a candidate threshold $z^{*}$. Then we show that, for any promised utility $z\left(s^{t-1}\right) \geq z^{*}$, there exists an allocation attaining the promised welfare $z\left(s^{t-1}\right)$ that satisfies all the incentive-compatibility constraints and features full insurance and no labor distortions. Finally we note that the proposed allocation solves an unconstrained optimization problem over continuation nodes, and thus must be part of the constrained efficient allocation.

For any $c^{e}>0$ let the system of functions $\left\{y^{e}\left(c^{e} ; \theta\right): \theta \in \Theta\right\}$ solve

$$
v^{\prime}\left(\frac{y^{e}\left(c^{e} ; \theta\right)}{\theta}\right)=u^{c}\left(c^{e}\right) \theta
$$

By Assumption 1 output $y^{e}\left(c^{e} ; \theta\right)$ is strictly decreasing with $c^{e}$. Let

$$
h^{e}\left(c^{e} ; \theta, \theta^{\prime}\right)=u\left(c^{e}\right)-v\left(\frac{y^{e}\left(c^{e} ; \theta^{\prime}\right)}{\theta}\right) .
$$

Clearly $h^{e}\left(c^{e} ; \theta, \theta\right)$ is increasing in $c^{e}$ as well for any $\theta$. Consider now the static payoff of misreporting $\theta^{\prime}$ when the true type is $\theta, h^{e}\left(c^{e} ; \theta, \theta^{\prime}\right)-h^{e}\left(c^{e} ; \theta, \theta\right)$. Since consumption is constant, the payoff is bounded above,

$$
h^{e}\left(c^{e} ; \theta, \theta^{\prime}\right)-h^{e}\left(c^{e} ; \theta, \theta\right) \leq v\left(\frac{y^{e}\left(c^{e} ; \theta\right)}{\theta}\right)
$$


The upper bound $v\left(\frac{y^{e}\left(c^{e} ; \theta\right)}{\theta}\right)$ is decreasing in $c^{e}$. Hence there exist $c^{*}$ such that for all $c^{e} \geq c^{*}$,

$$
p h^{e}\left(c^{e} ; \theta, \theta\right) \geq p \Upsilon+(1-p)\left(h^{e}\left(c^{e} ; \theta, \theta^{\prime}\right)-h^{e}\left(c^{e} ; \theta, \theta\right)\right)
$$

for all $\theta, \theta^{\prime}$. The candidate threshold is

$$
z^{*}=\frac{1}{1-\beta} \sum_{\theta \in \Theta} \pi(\theta) h^{e}\left(c^{*} ; \theta, \theta\right) .
$$

For any $z\left(s^{t-1}\right)$ there exists a consumption level $c^{e}$ such that

$$
z\left(s^{t-1}\right)=\frac{1-\beta^{T+1-t}}{1-\beta} \sum_{\theta \in \Theta} \pi(\theta) h^{e}\left(c^{e} ; \theta, \theta\right),
$$

since $h^{e}$ is increasing in $c^{e}$. Hence if $z\left(s^{t-1}\right) \geq z^{*}$ then the solution above satisfies $c^{e} \geq c^{*}$. Consider an allocation over the continuation nodes $\left\{s^{j} \in S^{j}: s^{t} \in s^{t-1}, j=t, t+1, \ldots, T\right\}$ with $c\left(s^{t}\right)=c^{e}$ and output levels as given by $y^{e}$. Note that the allocation prescribe the same continuation welfare for all types at a given date, $d=t, t+1, \ldots, T$,

$$
z_{d}^{e}=\frac{1-\beta^{T+1-d}}{1-\beta} \sum_{\theta \in \Theta} \pi(\theta) h^{e}\left(c^{e} ; \theta, \theta\right) .
$$

Now we show that such an allocation satisfies the incentive-compatibility constraints over all continuation nodes. Re-arrange terms in (16) for any pair $\theta, \theta^{\prime}$ and date $d=t, t+1, \ldots, T$ to obtain

$$
\begin{aligned}
h^{e}\left(c^{e} ; \theta, \theta\right) & \geq p \Upsilon+(1-p) h^{e}\left(c^{e} ; \theta, \theta^{\prime}\right) \\
& \geq p\left(1-\beta^{T+1-d}\right) \Upsilon+(1-p) h^{e}\left(c^{e} ; \theta, \theta^{\prime}\right)
\end{aligned}
$$

Adding $\beta z_{d+1}^{e}$ to both sides results in the incentive-compatibility constraint for pair $\theta, \theta^{\prime}$ and date $d$.

Finally, note that the proposed allocation over continuation nodes is the solution to the unconstrained problem:

$$
\min _{\{\hat{c}, \hat{y}\}} \sum_{j=t}^{T} \sum_{s^{j} \in S^{j} \mid s^{t-1}} R^{-(j-t)} \pi\left(s^{j}\right)\left\{\hat{c}\left(s^{j}\right)-\hat{y}\left(s^{j}\right)\right\}
$$


subject to

$$
\sum_{j=t}^{T} \sum_{s^{j} \in S^{j} \mid s^{t-1}} \beta^{(j-t)} \pi\left(s^{j}\right) h\left(\hat{c}\left(s^{j}\right), \hat{y}\left(s^{j}\right) ; \theta_{j}\right) \geq z\left(s^{t-1}\right)
$$

and non-negativity constraints. Thus the constrained efficient allocation must be equal to the proposed allocation over the continuation nodes

Proof: [Proof of Proposition 4] For any $c^{e}>0$ let the system of functions $\left\{y^{e}\left(c^{e} ; \theta\right): \theta \in \Theta\right\}$ solve

$$
v^{\prime}\left(\frac{y^{e}\left(c^{e} ; \theta\right)}{\theta}\right)=u^{c}\left(c^{e}\right) \theta
$$

and let

$$
h^{e}\left(c^{e} ; \theta\right)=u\left(c^{e}\right)-v\left(\frac{y^{e}\left(c^{e} ; \theta\right)}{\theta}\right) .
$$

Clearly $h^{e}\left(c^{e} ; \theta\right)$ is strictly increasing in $c^{e}$ for all $\theta$. Pick $\bar{c}$ such that

$$
\beta \pi(\bar{\theta}) h^{e}(\bar{c} ; \bar{\theta})=z^{*}-(1+\beta) \Upsilon
$$

where $z^{*}$ is the threshold value given by Proposition 3 .

Let $\{x, K\}$ be a constrained efficient allocation and assume that there exists $s^{t}$ such that $c\left(s^{t}\right)>\bar{c}$. We take $\theta_{t}=\bar{\theta}$ without loss of generality as $c\left(s^{t-1}, \bar{\theta}\right) \geq c\left(s^{t}\right)$ by the single-crossing condition. If $t \leq 1$, then (17) implies that $W_{0}(x) \geq z^{*}$ by construction. Proposition 3 then concludes that $\{x, K\}$ is efficient. If $t>1$, then (17) implies that $z\left(s^{t-2}\right) \geq z^{*}$ by construction. Proposition 3 then implies that $c\left(s^{t-1}\right)=c\left(s^{t}\right) \geq \bar{c}$. By induction, we conclude that $\{x, K\}$ is efficient. Thus if $\{x, K\}$ is not efficient then we must have $c\left(s^{t}\right) \leq \bar{c}$.

Propositions 6 and 4, together with the characterization for consumption

$$
u^{\prime}\left(s^{t}\right)\left(1+\eta\left(s^{t}\right)\right)=\lambda_{t},
$$

imply that the Lagrangian $\eta\left(s^{t}\right)$ is bounded above and below. We state this result as a simple Lemma that comes in use in Theorems 1 and 2.

\section{Lemma 3}

Let $p>0$ and $\{x, K\}$ be a constrained efficient allocation and $\left\{\eta\left(s^{t}\right): s^{t} \in S^{t}, t \geq 0\right\}$ the associated Lagrangian multiplier on the promise-keeping constraint (3). Then

$$
-1<B_{0} \leq \eta\left(s^{t}\right) \leq B_{1}<\infty
$$

for all $s^{t} \in S^{t}, t \geq 0$. 
Proof: The lower bound follows from Proposition 6. Since $c\left(s^{t}\right) \geq c^{b}>0$, f.o.n.c. (18) implies

$$
\eta\left(s^{t}\right)=\frac{\lambda}{u^{\prime}\left(c\left(s^{t}\right)\right)}-1 \geq \frac{\lambda}{\left.u^{\prime}\left(c^{b}\right)\right)}-1>-1 .
$$

For the upper bound we distinguish two cases. If $\{x, K\}$ is efficient then consumption is constant at all nodes, and by (18) it follows that $\eta\left(s^{t}\right)$ is also constant and finite. If $\{x, K\}$ is not efficient then $c\left(s^{t}\right) \leq \bar{c}<\infty$ by Proposition 4 . Thus by (18)

$$
\eta\left(s^{t}\right)=\frac{\lambda}{u^{\prime}\left(c\left(s^{t}\right)\right)}-1 \leq \frac{\lambda}{\left.u^{\prime}(\bar{c})\right)}<\infty
$$

\section{Proofs for Section 6}

Proof: [Proof of Theorem 1] The proof for has two parts. First we show that if

$$
u^{\prime}\left(s^{t}\right)<E_{t}\left\{u^{\prime}\left(s^{t+1}\right)\right\}
$$

at some node $s^{t}$, then for some pair $\theta, \theta^{\prime}$ we have $\mu\left(s^{t}, \theta, \theta^{\prime}\right) \geq \bar{\mu}>0$. For the second part we proceed by contradiction: if there does not exist a finite $T$ such that $u^{\prime}\left(s^{t}\right) \geq E_{t}\left\{u^{\prime}\left(s^{t+1}\right)\right\}$ for some node, then $\eta\left(s^{t}\right)$ would violate the upper bound imposed by Lemma 3 .

Let the intertemporal wedge be given by

$$
\tau\left(s^{t}\right)=1-\frac{u^{\prime}\left(s^{t}\right)}{E\left[u^{\prime}\left(s^{t+1}\right)\right]} .
$$

Using the f.o.n.c. we have

$$
\begin{aligned}
\tau\left(s^{t}\right) & =1-\frac{1+\frac{p \mu^{*}\left(s^{t}\right)}{1+\eta\left(s^{t}\right)}}{E\left[u^{\prime}\left(s^{t+1}\right)\right] E\left[\frac{1}{u^{\prime}\left(s^{t+1}\right)}\right]} \\
& =1-\frac{\frac{\lambda_{t}}{1+\eta\left(s^{t}\right)}}{E\left[u^{\prime}\left(s^{t+1}\right)\right]} .
\end{aligned}
$$

We differentiate the wedge with respect to some $\mu\left(s^{t}, \theta, \theta^{\prime}\right)$ :

$$
\frac{\partial \tau}{\partial \mu\left(s^{t}, \theta, \theta^{\prime}\right)}=\frac{\frac{\lambda_{t}}{1+\eta\left(s^{t}\right)}}{\beta F_{t+1}^{K}\left(E\left[u^{\prime}\left(s^{t+1}\right)\right]\right)^{2}} \cdot \frac{\partial E\left[u^{\prime}\left(s^{t+1}\right)\right]}{\partial \mu\left(s^{t}, \theta, \theta^{\prime}\right)}
$$


Using the f.o.n.c, we write the expected marginal utility as

$$
\begin{aligned}
\frac{E\left[u^{\prime}\left(s^{t+1}\right)\right]}{\lambda_{t+1}} & =p \sum_{\theta} \frac{\pi(\theta)}{1+\eta\left(s^{t}\right)+\sum_{\theta^{\prime}} \mu\left(s^{t}, \theta, \theta^{\prime}\right)} \\
& +(1-p) \sum_{\theta} \frac{\pi(\theta)}{1+\eta\left(s^{t}\right)+\sum_{\theta^{\prime}}\left\{\mu\left(s^{t}, \theta, \theta^{\prime}\right)-\frac{\pi\left(\theta^{\prime}\right)}{\pi(\theta)} \mu\left(s^{t}, \theta^{\prime}, \theta\right)\right\}} .
\end{aligned}
$$

For any node $s^{t}$ with $t<T$, and pair of types $\theta_{i}, \theta_{j}$ we can compute the derivative as

$$
\begin{aligned}
\frac{\partial E\left[u^{\prime}\left(s^{t+1}\right)\right]}{\partial \mu\left(s^{t}, \theta_{i}, \theta_{j}\right)} & =-p \frac{\lambda_{t+1} \pi\left(\theta_{i}\right)}{\left(1+\eta\left(s^{t}\right)+\sum_{\theta^{\prime}} \mu\left(s^{t}, \theta_{i}, \theta^{\prime}\right)\right)^{2}} \\
& -\frac{(1-p) \lambda_{t+1} \pi\left(\theta_{i}\right)}{\left(1+\eta\left(s^{t}\right)+\sum_{\theta^{\prime}}\left\{\mu\left(s^{t}, \theta_{i}, \theta^{\prime}\right)-\frac{\pi\left(\theta^{\prime}\right)}{\pi\left(\theta_{i}\right)} \mu\left(s^{t}, \theta^{\prime}, \theta_{i}\right)\right\}\right)^{2}} \\
& +\frac{(1-p) \lambda_{t+1} \pi\left(\theta_{i}\right)}{\left(1+\eta\left(s^{t}\right)+\sum_{\theta^{\prime}}\left\{\mu\left(s^{t}, \theta_{j}, \theta^{\prime}\right)-\frac{\pi\left(\theta^{\prime}\right)}{\pi\left(\theta_{j}\right)} \mu\left(s^{t}, \theta^{\prime}, \theta_{j}\right)\right\}\right)^{2}} .
\end{aligned}
$$

We evaluate $\frac{\partial E\left[u^{\prime}\left(s^{t+1}\right)\right]}{\partial \mu\left(s^{t}, \theta_{i}, \theta_{j}\right)}$ at $\vec{\mu}=0$ to obtain

$$
\left.\frac{\partial E\left[u^{\prime}\left(s^{t+1}\right)\right]}{\partial \mu\left(s^{t}, \theta_{i}, \theta_{j}\right)}\right|_{\vec{\mu}=0}=-p \frac{\lambda_{t+1} \pi\left(\theta_{i}\right)}{\left(1+\eta\left(s^{t}\right)\right)^{2}} .
$$

By Lemma 3 multiplier $\eta\left(s^{t}\right)$ is bounded above by $B_{\eta}<\infty$. Hence

$$
\left.\frac{\partial E\left[u^{\prime}\left(s^{t+1}\right)\right]}{\partial \mu\left(s^{t}, \theta_{i}, \theta_{j}\right)}\right|_{\vec{\mu}=0} \leq-p \frac{\lambda \pi\left(\theta_{i}\right)}{\left(1+B_{\eta}\right)^{2}}<0 .
$$

It follows that the intertemporal wedge is negative $\tau\left(s^{t}\right)<0$ if for all pairs $\theta_{i}, \theta_{j}$ we have $\mu\left(s^{t}, \theta_{i}, \theta_{j}\right)<\bar{\mu}$, where $\bar{\mu}$ is strictly positive and constant across nodes and valid for all horizons $T$.

For a given horizon $T$ and constrained-efficient allocation assume that at all nodes for dates $t<T$ we have $\tau\left(s^{t}\right)>0$. We can then construct a type $\hat{s}^{T}$ by selecting such pair and $a_{t}=1$ for all dates, that is, for any node $\hat{s}^{t}$ select $\hat{s}^{t+1}=\left\{\hat{s}^{t}, \theta, 1\right\}$ where $\theta$ is such that $\mu\left(s^{t}, \theta, \theta^{\prime}\right) \geq \bar{\mu}$ for some $\theta^{\prime}$. If such $\theta$ does not exist, then $\tau\left(s^{t}\right) \leq 0$ contradicting the premise.

By f.o.n.c. 14 we have that

$$
\eta\left(\hat{s}^{T}\right) \geq T \bar{\mu} .
$$

If there does not exist a finite $T$ such that $\tau\left(s^{t}\right) \leq 0$ for some node, then (19) implies that there exists a finite $T^{*}$ with $T^{*} \bar{\mu}>B_{\eta}$, contradicting Lemma 3 . 


\section{Proofs for Section 7}

Proof: [Proof of Lemma 2] Let $\bar{\theta}=\max \{\theta \in \Theta\}$ and $x$ a constrained efficient allocation with $z$ the associated plan of continuation welfare. We show that for all nodes $s^{t}$,

$$
h\left(x\left(s^{t}\right) ; \bar{\theta}\right)+\beta z\left(s^{t}\right)>\Upsilon .
$$

As previously shown, allocations for the top skill agent do not depend on the realization of $a_{t}$ and we can write the system of incentive-compatibility constraints for such agent as

$$
h\left(x\left(s^{t}\right) ; \bar{\theta}\right)+\beta z\left(s^{t}\right) \geq p \Upsilon+(1-p)\left(h\left(x\left(\hat{s}^{t}\right) ; \bar{\theta}\right)+\beta z\left(\hat{s}^{t}\right)\right)
$$

for any $\theta^{\prime} \neq \bar{\theta}, \hat{s}^{t}=\left\{s^{t-1}, 0, \theta^{\prime}\right\}$. By Proposition $6, y\left(\hat{s}^{t}\right)>0$ and thus

$$
h\left(x\left(\hat{s}^{t}\right) ; \bar{\theta}\right)>h\left(x\left(\hat{s}^{t}\right) ; \theta^{\prime}\right) .
$$

The lower bound on welfare (1) implies

$$
h\left(x\left(\hat{s}^{t}\right) ; \theta^{\prime}\right)+\beta z\left(\hat{s}^{t}\right) \geq \Upsilon,
$$

and thus (20) follows. Since $\pi\left(\theta_{t+1}=\bar{\theta} \mid s^{t}\right)>0$ it is immediate that $z\left(s^{t}\right)>\Upsilon$.

Proof: [Proof of Theorem 2] Define

$$
\tilde{\eta}_{t} \equiv E\left\{\eta\left(s^{t}\right)\right\}=\sum_{s^{t} \in S^{t}} \pi\left(s^{t}\right) \eta\left(s^{t}\right)
$$

By the law of iterated expectations and the conditional expectation of $\eta_{t+1}$, we have that

$$
\tilde{\eta}_{t+1}=\tilde{\eta}_{t}+p E\left\{\mu^{*}\left(s^{t}\right)\right\}
$$

Since $\mu^{*}\left(s^{t}\right) \geq 0$ the sequence $\left\{\tilde{\eta}_{t}\right\}$ is increasing by $(21)$. Because the sequence is bounded above, it necessarily has to converge. Thus $\mu^{*}\left(s^{t}\right)$ converges in mean to 0 ,

$$
\lim _{t \rightarrow \infty} \sum_{s^{t} \in S^{t}} \pi\left(s^{t}\right) \mu^{*}\left(s^{t}\right)=0
$$


Since all terms are positive,

$$
\lim _{t \rightarrow \infty} \sum_{s^{t} \in S^{t}} \pi\left(s^{t}\right) \mu\left(s^{t}, \theta ; \theta^{\prime}\right)=0
$$

for all $\theta, \theta^{\prime} \in \Theta$. Convergence-in-mean implies convergence-in-probability, so for any $\varepsilon>0$, the probability $\operatorname{Pr}\left(\mu\left(s^{t}, \theta ; \theta^{\prime}\right)>\varepsilon\right)$ converges to 0 for all $\theta, \theta^{\prime} \in \Theta$.

Next we show that if $\left|c\left(s^{t}\right)-c\left(s^{t+1}\right)\right| \geq \delta$ for some $\delta>0$ then $\mu\left(s^{t}, \theta ; \theta^{\prime}\right) \geq \varepsilon_{\delta}>0$ for some pair $\left\{\theta, \theta^{\prime}\right\}$. Since we have shown the Lagrange multiplier on promised utility $\eta_{t}$ to be bounded, $c\left(s^{t}\right)$ is bounded above and below, and $\kappa=\inf \left\{\left|u^{\prime \prime}\left(s^{t}\right) /\left(u^{\prime}\left(s^{t}\right)\right)^{2}\right|: s^{t} \in S^{t}, t \geq 0\right\}$ is finite. Using a Taylor expansion we have that

$$
\begin{aligned}
\left|\frac{\lambda}{u^{\prime}\left(s^{t}\right)}-\frac{\lambda}{u^{\prime}\left(s^{t+1}\right)}\right| & \geq \kappa\left|c\left(s^{t}\right)-c\left(s^{t+1}\right)\right| \\
& \geq \kappa \delta=\delta^{\prime} .
\end{aligned}
$$

If $a_{t+1}=0$ then (9) at $s^{t+1}$ combined with (18) at $s^{t}$ imply

$$
\left|\sum_{\theta^{\prime} \in \Theta}\left\{\mu\left(s^{t}, \theta_{t+1} ; \theta^{\prime}\right)-\frac{\pi\left(\theta^{\prime}\right)}{\pi\left(\theta_{t+1}\right)} \mu\left(s^{t}, \theta^{\prime} ; \theta_{t+1}\right)\right\}\right| \geq \delta
$$

and thus

$$
\sum_{\theta^{\prime} \in \Theta}\left\{\left|\mu\left(s^{t}, \theta_{t+1} ; \theta^{\prime}\right)\right|+\frac{1}{\pi\left(\theta_{t+1}\right)}\left|\mu\left(s^{t}, \theta^{\prime} ; \theta_{t+1}\right)\right|\right\} \geq \delta^{\prime} .
$$

Set

$$
\varepsilon_{\delta}=\frac{\delta^{\prime} \min \{\pi(\theta): \theta \in \Theta\}}{2 N_{\Theta}}
$$

where $N_{\Theta}$ is the cardinality of set $\Theta$. By construction, if $\mu\left(s^{t}, \theta ; \theta^{\prime}\right)<\varepsilon_{\delta}$ for all $\left\{\theta, \theta^{\prime}\right\}$ then we contradict $(22)$.

If $a_{t+1}=1$ then (12) at $s^{t+1}$ combined with (18) at $s^{t}$ imply

$$
\left|\sum_{\theta^{\prime} \in \Theta} \mu\left(s^{t}, \theta_{t+1} ; \theta^{\prime}\right)\right| \geq \delta^{\prime}
$$

and thus $\mu\left(s^{t}, \theta ; \theta^{\prime}\right)>\varepsilon_{\delta}$ for some $\left\{\theta, \theta^{\prime}\right\}$.

Thus for any $\delta>0$

$$
\lim _{t \rightarrow \infty} \operatorname{Pr}\left(\left|c\left(s^{t}\right)-c\left(s^{t+1}\right)\right| \geq \delta\right) \leq \lim _{t \rightarrow \infty} \operatorname{Pr}\left(\mu\left(s^{t}, \theta ; \theta^{\prime}\right) \geq \varepsilon_{\delta}\right)=0 .
$$

Next we show that there are no labor distortions in the limit following very similar steps. If 
$\left|u^{\prime}\left(s^{t}\right)-h^{y}\left(s^{t} ; \theta_{t}\right)\right| \geq \delta$ then by manipulation of the f.o.n.c. (10) we have that

$$
\left|u^{\prime}\left(s^{t}\right)-h^{y}\left(s^{t} ; \theta_{t}\right)\right|=\frac{\sum_{\theta^{\prime} \in \Theta} \frac{\pi\left(\theta^{\prime}\right)}{\pi\left(\theta_{t}\right)} \mu\left(s^{t-1}, \theta^{\prime} ; \theta_{t}\right) h^{y}\left(s^{t} ; \theta^{\prime}\right)}{1+\eta\left(s^{t}\right)} .
$$

For all binding IC constraints, $h^{y}\left(s^{t} ; \theta^{\prime}\right) \leq h^{y}\left(s^{t} ; \theta_{t}\right)$, and with the first-order condtions for the top-skill agent and $2, h^{y}\left(s^{t} ; \theta_{t}\right) \leq u^{\prime}\left(s^{t}\right)$. Furthermore, Lemma 3 implies $u^{\prime}\left(s^{t}\right) \leq \kappa<\infty$ and $-1<B_{0} \leq \eta\left(s^{t}\right)$, so

$$
\left|u^{\prime}\left(s^{t}\right)-h^{y}\left(s^{t} ; \theta_{t}\right)\right| \leq \frac{1}{\left(1+B_{0}\right) \pi\left(\theta_{t}\right)} \sum_{\theta^{\prime} \in \Theta} \mu\left(s^{t-1}, \theta^{\prime} ; \theta_{t}\right) \kappa
$$

By setting $\varepsilon_{\delta}=\delta\left(1+B_{0}\right) \min \{\pi(\theta): \theta \in \Theta\} /\left(\kappa N_{\Theta}\right)$ we can show that if $\left|u^{\prime}\left(s^{t}\right)-h^{y}\left(s^{t} ; \theta_{t}\right)\right| \geq \delta$ then $\mu\left(s^{t}, \theta ; \theta^{\prime}\right)>\varepsilon_{\delta}$ for some $\left\{\theta, \theta^{\prime}\right\}$ and $\varepsilon_{\delta}>0$. Hence

$$
\lim _{t \rightarrow \infty} \operatorname{Pr}\left(\left|u^{\prime}\left(s^{t}\right)-h^{y}\left(s^{t} ; \theta_{t}\right)\right| \geq \delta\right)=0
$$

for any $\delta>0$. 


\section{References}

Albanesi, Stefania and Christopher Sleet, "Dynamic Optimal Taxation with Private Information," The Review of Economic Studies, 2006, 73, 1-30.

Atkeson, Andrew and Robert E. Lucas, "On Efficient Distribution with Private Information," The Review of Economic Studies, 1993, 59 (3), 427-453.

Border, Kim C. and Joel Sobel, "Samurai Accountant: A Theory of Auditing and Plunder," The Review of Economic Studies, 1987, 54 (4), 525-540.

Chamley, Christophe, "Optimal Taxation of Capital Income in General Equilibrium with Infinite Lives," Econometrica, 1986, 54 (3), 607-622.

Cremer, Helmuth and Firouz Gahvari, "Tax evasion and the optimum general income tax," Journal of Public Economics, 1995, 60, 235-249.

Farhi, Emmanuel and Ivan Werning, "Capital Taxation: Quantitative explorations of the inverse Euler equation," MIT Working Paper 06-15, 2007.

Golosov, Mikhail and Aleh Tsyvinski, "Designing Optimal Disability Insurance: A Case for Asset Testing," Journal of Political Economy, 2006, 114 (2), 257-279.

_, Narayana Kocherlakota, and Aleh Tsyvinski, "Optimal Indirect and Capital Taxation," The Review of Economic Studies, 2003, 70 (3), 569-587.

Judd, Kenneth L., "Redistributive Taxation in a Perfect Foresight Model," Journal of Public Economics, 1985, 28, 59-84.

Kocherlakota, Narayana, "Expected Wealth Taxes: A Mirrlees Approach to Dynamic Optimal Taxation," Econometrica, 2005, 73 (5), 1587-1621.

Mirrlees, James, "An exploration in the theory of optimum income taxation," The Review of Economic Studies, 1971, 38, 175-208.

Rogerson, William, "Repeated moral hazard," Econometrica, 1985, 53, 69-76.

Salanie, Bernard, The economics of Taxation, The MIT Press, Cambridge, MA, 2003.

Stokey, Nancy and Robert Lucas, Recursive Methods in Economic Dynamics, Harvard University Press, Cambridge, MA, 1996.

Tuomala, Matti, Optimal Income Tax and Redistribution, Oxford University Press, 1990.

Werning, Ivan, "Optimal Fiscal Policy with Redistribution," The Quarterly Journal of Economics, 2007, 122 (3), 925-967. 